ARTICLE

Received 1 Feb 2016 | Accepted 4 May 2016 | Published 17 Jun 2016

DOI: $10.1038 /$ ncomms11841

OPEN

\title{
A threshold level of NFATc1 activity facilitates thymocyte differentiation and opposes notch- driven leukaemia development
}

\author{
Stefan Klein-Hessling ${ }^{1}$, Ronald Rudolf ${ }^{1}$, Khalid Muhammad ${ }^{1}$, Klaus-Peter Knobeloch², \\ Muhammad Ahmad Maqbool ${ }^{3}$, Pierre Cauchy ${ }^{4}$, Jean-Christophe Andrau ${ }^{3}$, Andris Avots ${ }^{1}$, \\ Claudio Talora ${ }^{5}$, Volker Ellenrieder ${ }^{6}$, Isabella Screpanti ${ }^{5}$, Edgar Serfling ${ }^{1}$ \& Amiya Kumar Patra ${ }^{1, \dagger}$
}

NFATc1 plays a critical role in double-negative thymocyte survival and differentiation. However, the signals that regulate Nfatcl expression are incompletely characterized. Here we show a developmental stage-specific differential expression pattern of Nfatc1 driven by the distal (P1) or proximal (P2) promoters in thymocytes. Whereas, preTCR-negative thymocytes exhibit only P2 promoter-derived $N$ fatc $1 \beta$ expression, preTCR-positive thymocytes express both $N f a t c 1 \beta$ and P1 promoter-derived Nfatcl $\alpha$ transcripts. Inducing NFATc1 $\alpha$ activity from P1 promoter in preTCR-negative thymocytes, in addition to the NFATc1 $\beta$ from P2 promoter impairs thymocyte development resulting in severe T-cell lymphopenia. In addition, we show that NFATC1 activity suppresses the B-lineage potential of immature thymocytes, and consolidates their differentiation to T cells. Further, in the pTCR-positive DN3 cells, a threshold level of NFATc1 activity is vital in facilitating T-cell differentiation and to prevent Notch3-induced T-acute lymphoblastic leukaemia. Altogether, our results show NFATc1 activity is crucial in determining the T-cell fate of thymocytes.

\footnotetext{
${ }^{1}$ Department of Molecular Pathology, Institute for Pathology, University of Wuerzburg, Josef-Schneider Strasse 2, 97080 Wuerzburg, Germany. ${ }^{2}$ Department of Pathology, Institute of Neuropathology, University Clinic Freiburg, Breisacher Strasse 64, 79106 Freiburg, Germany. ${ }^{3}$ Institute de Génétique Moléculaire de Montpellier (IGMM), UMR5535 CNRS, 1919 Route de Mende, 34293 Montpellier Cedex 5, France. ${ }^{4}$ Institute of Biomedical Research, College of Medical and Dental Sciences, University of Birmingham, Birmingham B15 2TT, UK. ${ }^{5}$ Laboratory of Molecular Pathology, Department of Molecular Medicine, Sapienza University of Rome, Viale Regina Elena 291, 00161 Rome, Italy. ${ }^{6}$ Department of Gastroenterology II, University of Goettingen, Robert Koch Strasse 40, 37075 Goettingen, Germany. † Present address: Institute of Translational and Stratified Medicine Peninsula Schools of Medicine and Dentistry, University of Plymouth, Plymouth Science Park, Research Way, Plymouth PL6 8BU, UK. Correspondence and requests for materials should be addressed to A.K.P. (email: amiya.patra@uni-wuerzburg.de).
} 
$\mathrm{D}$ ifferentiation of $\mathrm{CD}^{-}{ }^{-} \mathrm{CD} 8{ }^{-}$double-negative (DN) cells to the $\mathrm{CD} 4{ }^{+}$or $\mathrm{CD} 8{ }^{+}$single-positive (SP) $\mathrm{T}$ cells in the thymus is regulated by a complex network of signalling pathways involving multiple transcription factors at various stages of development. On the basis of their differentiation status, DN thymocytes consist of four distinct populations, CD $44^{+}$ CD25 ${ }^{-} \mathrm{DN} 1, \mathrm{CD}_{4} 4^{+} \mathrm{CD} 25^{+} \mathrm{DN} 2, \mathrm{CD} 44^{-} \mathrm{CD} 25^{+} \mathrm{DN} 3$ and $\mathrm{CD} 44^{-} \mathrm{CD} 25^{-} \mathrm{DN} 4$ (ref. 1). DN3 thymocytes upon rearrangement of their T-cell receptor $\beta$ (Tcrb) locus express a functional $\mathrm{TCR} \beta$ chain, which, in combination with the $\operatorname{preT} \alpha(\mathrm{pT} \alpha)$ chain forms the preTCR (pTCR). PreTCR signalling is essential for the differentiation of DN3 cells to DN4 and later stages. We have shown recently that the transcription factor NFATc1 plays a critical role during early stages of thymocyte development ${ }^{2}$. A haematopoietic lineage cell-specific ablation of NFATc1 activity blocked thymocyte development at DN1 stage, the earliest stage of thymic T-cell development ${ }^{2}$. Mice deficient for NFATc2, NFATc3 or both, do not show any apparent defect in thymocyte development ${ }^{3,4}$, suggesting a critical role played by NFATc1 during thymic T-cell development.

Ligation of TCR with cognate peptide-MHC complex in vivo, or stimulation of $\mathrm{T}$ cells with anti-CD3 plus anti-CD28 Abs in vitro increases intracellular $\mathrm{Ca}^{2}+$ levels, which in turn activate the serine/threonine phosphatase calcineurin. Active calcineurin dephosphorylates multiple serine/threonine residues in NFAT proteins and facilitates their nuclear translocation. We have previously elucidated a novel NFAT activation pathway in pTCR-negative thymocytes, which plays an indispensable role in their survival and differentiation ${ }^{2}$. In contrast to the calcineurin-mediated dephosphorylation pathway, in pTCRnegative thymocytes IL-7-JAK3 signals activated NFATc1 via phosphorylation of tyrosine ${ }^{371}$ in the regulatory domain ${ }^{2}$. Both, the calcineurin-mediated 'conventional' and IL-7-JAK3-mediated 'alternative' pathways though explained the post-translational mechanisms of NFAT activation, the transcriptional regulation of Nfatcl itself is poorly understood. A previous study showed Nfatcl expression in T cells is autoregulated by NFATc1 (ref. 5). In this study, we have delineated the signalling pathways that regulate $\mathrm{Nfatcl}$ expression with distinct promoter usage at pTCRnegative and -positive thymocytes. Further, we provide evidence in support of a critical role of NFATcl in suppressing lineage plasticity of immature thymocytes towards non-T lineages, and the essentiality of a threshold level of NFATc1 activity at the pTCR-positive DN3 stage in facilitating the T-cell fate of thymocytes by preventing the development of T-Acute Lymphoblastic Leukaemia (T-ALL).

\section{Results}

Differential usage of Nfatc1 promoters in thymocytes. In $\mathrm{T}$ cells, two distinct promoters, a distal (P1) and a proximal (P2), initiate $N f a t c 1$ expression. Due to alternative splicing and usage of two different poly-adenylation (pA) sites, six Nfatcl isoforms; three from P1 promoter (Nfatcl $\alpha \mathrm{A}, \alpha \mathrm{B}$ and $\alpha \mathrm{C})$ and three from $\mathrm{P} 2$ promoter (Nfatc1 $\beta \mathrm{A}, \beta \mathrm{B}$ and $\beta \mathrm{C})$, respectively, are synthesized (Supplementary Fig. 1a) ${ }^{6,7}$. To distinguish whether any particular NFATcl isoform is prevalent in pTCR-negative and -positive thymocytes we analysed wild-type (WT) DN1-DN4 cells for Nfatcl expression. Interestingly, we observed an exclusive P2 promoter activity in the pTCR-negative DN3 cells, whereas pTCR-positive DN4 cells exhibited both P2 as well as P1 promoter activity (Fig. 1a; Supplementary Fig. 1b). Exclusive P2 activity in pTCR-negative thymocytes was supported by an active chromatin configuration, as indicated by histone modifications and a concomitant recruitment of RNA polymerase II (Pol II) at the Nfatc1 P2 promoter in Rag2 ${ }^{-1-}$ DN3 cells, whereas only little H3K4me3 was detectable at the P1 promoter (Fig. 1b). Analysis of small RNA-seq data ${ }^{8}$, to score for bidirectional paused transcripts enriched at promoters further confirmed significant enrichment of transcriptional start site RNAs at P2 promoter over P1 (Fig. 1b). Using an additional set of primers to detect total $\alpha$ - or $\beta$-specific transcripts derived from the P1 or P2 promoters respectively, again we observed an exclusive P2 activity in DN3, and both P1 and P2 activity in DN4 cells (Supplementary Fig. 1c). Further, in agreement with our observation regarding induction of $\mathrm{P} 1$ promoter activity at the PTCR-positive stages, we detected NFATc1 $\alpha$ proteins in DN4 cells but not in DN3 cells (Fig. 1c; Supplementary Fig. 1d). Keeping in view the findings of a previous report ${ }^{5}$, that in naive T cells, constitutive basal level TCR signalling maintains the $\mathrm{P} 2$ promoter activity, and TCR-antigen ligation induced signals are responsible for the P1 promoter activity, it was quite intriguing how thymocytes lacking even the pTCR exhibit such a robust Nfatc1 expression.

To further substantiate our observation regarding selective promoter usage, and the role of pTCR signalling in inducing P1 activity, we investigated three different mouse models, where either there was no pTCR $\left(R a g 1^{-1-}\right)$, pTCR was absent but a downstream signalling molecule calcineurin was constitutively active (Calcineurin transgenic; $\Delta \mathrm{Cam})^{9}$ or there was enhanced pTCR signalling (Notch3 transgenic; N3 tg) ${ }^{10}$. In both Rag1 ${ }^{-1-}$ and $\Delta$ Cam mice, T-cell development is blocked at the DN3 stage due to lack of pTCR signals ${ }^{9}$. In contrast, N3 tg mice showed an enhanced DN3 to DN4 transition due to strong pTCR signals (Fig. 1d,e). Confirming our ChIP-Seq and RNA-Seq observations, analysis of $\mathrm{Nfatc1}$ expression in Rag1 ${ }^{-1-} \mathrm{DN} 3$ cells showed the presence of only Nfatc1 $\beta$ isoforms (P2 activity), similar to that in WT DN3 cells (Fig. 1f). Stimulating Rag1 ${ }^{-l-}$ DN3 cells with anti-CD3 Abs to mimic pTCR signalling, we readily detected $N f a t c 1 \alpha$ transcripts in addition to the $N f a t c 1 \beta$ transcripts implying that pTCR signals can induce P1 promoter activity (Fig. 1f). Further, P1 promoter activity was autoregulated by NFATc1, as P1 activity disappeared without affecting P2 activity when anti-CD3 antibodies stimulated Rag1 ${ }^{-1-}$ DN3 cells were treated with cyclosporine A (CsA; Fig. 1f). In contrast to the WT and Rag1 ${ }^{-1-}$ mice, $\Delta$ Cam DN3 cells showed a robust P1 activity in addition to the P2 promoter activity (Fig. 1g). The strong P1 activity in $\triangle$ Cam DN3 cells was due to the autoregulatory loop maintained by constitutive calcineurin activity, as CsA treatment specifically extinguished $\mathrm{Nfatc1}$ P1 activity without affecting the P2 activity (Fig. 1g). Further, we observed a similar pattern of strong $\mathrm{P} 1$ and $\mathrm{P} 2$ promoter activity as that in $\Delta$ Cam mice, in N3 tg DN3 cells (Fig. 1h). Thus, it was evident that Nfatc1 is expressed from distinct promoters in pTCR-negative and -positive thymocytes, and PTCR signalling is necessary for the induction of Nfatc1 P1 promoter activity.

NFATc1 activity is vital for DN thymocyte differentiation. Due to exclusive P2 activity in PTCR-negative thymocytes, we investigated whether NFATc1 $\beta$ is solely critical for the differentiation of early DN thymocytes. To clarify this, we have generated a mutant mouse with floxed $N f a t c 1$ P2 promoter element $\left(P 2^{\mathrm{fl} / \mathrm{fl}}\right)$ to abolish NFATc1 $\beta$ activity in a tissue-specific manner (Supplementary Fig. 2a-d). We bred $P 2^{\mathrm{fl} / \mathrm{fl}}$ mice with mice expressing cre-recombinase under $\operatorname{Vav}$ promoter $(\operatorname{Vav} \text {-Cre })^{11}$ to abolish Nfatc1 P2 activity during thymocyte development. Surprisingly, analysis of $V a v$-CreP2 $2^{\mathrm{f} / f l}$ mice showed completely normal T-cell development, which was indistinguishable from that of littermate controls (Fig. 2a-c; Supplementary Fig. 2e). The normal T-cell development in $\mathrm{Vav}$-CreP2 $2^{\mathrm{f} / \mathrm{fl}}$ mice was quite surprising, as loss of NFATc1 activity was expected to block thymocyte development at the DN1 stage ${ }^{2}$. PCR with reverse 
a

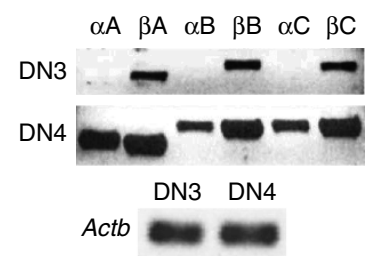

C

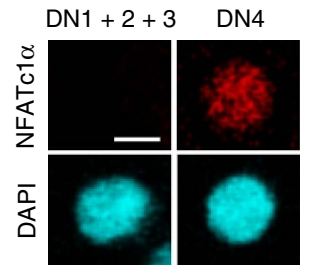

b $\mathrm{P} 1 \mathrm{P} 2$

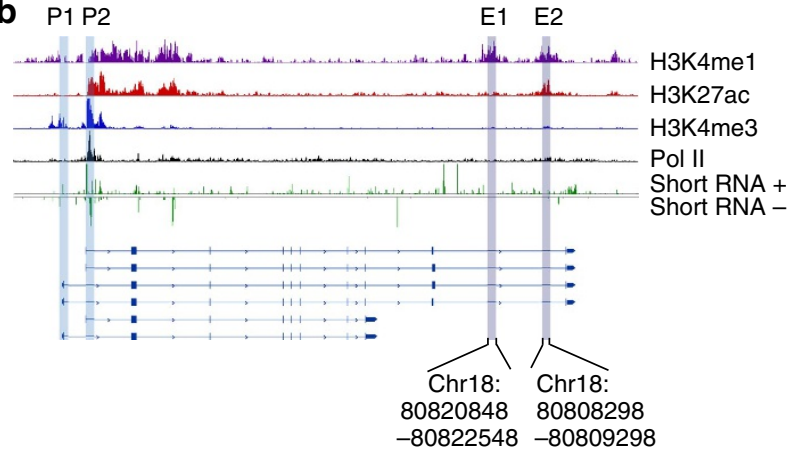

d

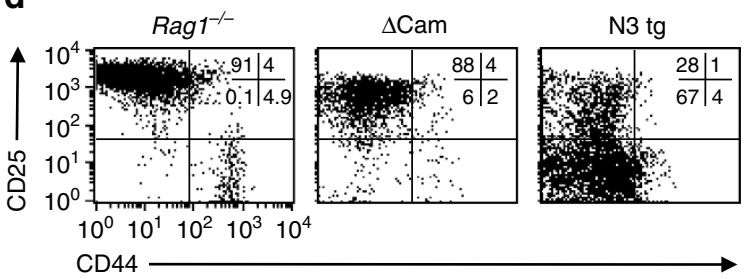

e

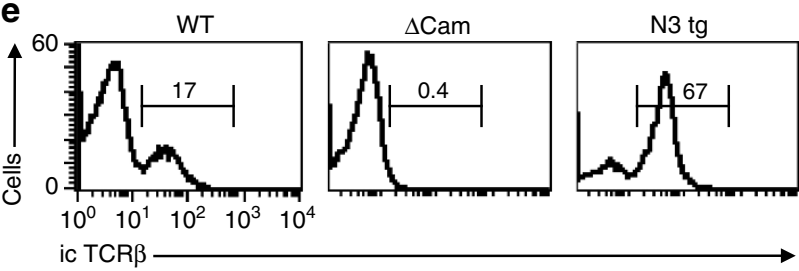

f

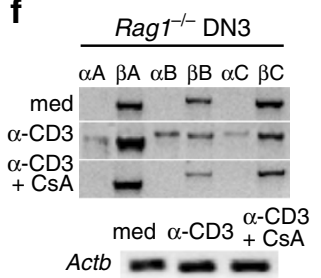

g

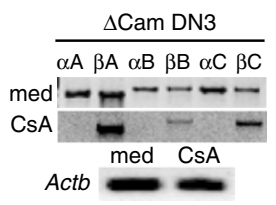

h

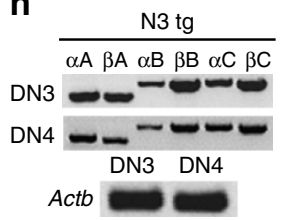

Figure 1 | Differential induction of Nfatc1 $\mathbf{P 1}$ and $\mathbf{P 2}$ promoters in pTCR-negative and -positive thymocytes. (a) RT-PCR analysis of Nfatc1 isoforms expression in WT DN3 and DN4 cells. (b) ChIP-Seq and small RNA-Seq analysis of Nfatc1 gene for histone methylation, acetylation, RNA Polymerase II binding and bidirectional transcriptional start site RNA at the P1 and P2 promoter region in Rag2-/- DN3 cells. Blue rectangles highlight promoters, whereas pink rectangles show putative enhancer hallmarks based on the presence of epigenetic/transcriptional features. Chromosomal coordinates represented below are the ones corresponding to DNase I hypersensitive sites described in Fig. 6a that are both inside the highlighted areas.

(c) Immunofluorescence analysis of NFATc1 $\alpha$ distribution in WT DN3 and DN4 cells. Nuclear staining was revealed by DAPI. Scale bar, $10 \mu \mathrm{m}$.

(d) Flow cytometry profiles of DN1 to DN4 cells distribution in indicated mice. (e) Intracellular TCR $\beta$ staining in WT, $\triangle$ Cam and N3 tg DN3 cells.

(f) RT-PCR analysis reveals the expression of six Nfatc1 isoforms in DN3 cells from Rag1 ${ }^{-1-}$ mice either freshly sorted, or treated with anti-CD3 Abs in absence or presence of $\mathrm{CsA}$ for $12 \mathrm{~h}$. (g) Transcript levels of six Nfatc1 isoforms in freshly isolated or $12 \mathrm{~h}$ CsA-treated DN3 cells from $\Delta$ Cam mice.

(h) Pattern of Nfatc1 isoforms expression in freshly sorted DN3 and DN4 cells from N3 tg mice. Numbers within each dot plot or histogram represent per cent respective population. Data are representative of three independent experiments.

transcription (RT-PCR) analysis on pTCR-negative thymocytes showed a total lack of Nfatc1 P2-derived transcripts indicating abolition of Nfatc1 P2 promoter activity (Fig. 2d). However, NFATc1 activity was not lost rather in absence of P2 activity we observed a robust P1 promoter activity in the $V a v-C r e P 2^{\mathrm{f} / / \mathrm{fl}}$ pTCR-negative thymocytes (Fig. 2d).

We have previously shown that Bcl-2 is a target of NFATc1 in pTCR-negative thymocytes ${ }^{2}$. However, analysis in $V a v$-CreP2 $2^{\mathrm{f} / f \mathrm{f}}$ DN3 cells showed similar levels Bcl-2 proteins as that in littermate controls (Supplementary Fig. 2f), suggesting that most likely NFATc1 $\alpha$ and $-\beta$ isoforms regulate a similar set of target genes that are critical for early thymocyte survival and differentiation. Thus, in the $V a v$-CreP2 $2^{\mathrm{fl} / \mathrm{ll}}$ mice, Nfatcl P1 promoter activity functionally compensated for the loss of P2 activity, underlining the indispensability of NFATcl activity in thymocyte differentiation.

NFATc1 activity is essential for T-cell development. To investigate the physiological significance of the distinct pattern of NFATc1 promoter activity in pTCR-negative and -positive thymocytes, we explored what impact NFATc1 $\alpha$ will have on thymocyte development if it is co-expressed with NFATc1 $\beta$ in pTCR-negative thymocytes. To address this issue, we used mice in which a constitutively active version of $N f a t c 1 \alpha A$ was knockedin into Rosa-26 locus flanked by a floxed stop cassette $\left(\text { R26-caNfatcl } \alpha \mathrm{A}-S \text { Stop } \mathrm{fl}^{\mathrm{f} / \mathrm{fl}} \text { : designated hereafter as } N f a t c 1 \alpha \mathrm{A}^{\mathrm{fl} / \mathrm{fl}}\right)^{12}$. To activate $N f a t c l \alpha A$ expression in early thymocytes we bred $N f a t c 1 \alpha \mathrm{A}^{\mathrm{f} / / \mathrm{fl}}$ mice with Vav-Cre mice. Surprisingly, analysis of Vav-CreNfatcl $\alpha \mathrm{A}^{\mathrm{f} / \mathrm{fl}}$ mice showed severely impaired thymocyte development as evident from a dose-dependent reduction in the size of the thymus, spleen and lymph nodes (Fig. 3a). Accordingly, the cellularity in these organs was drastically reduced in $V a v$-CreNfatcl $\alpha \mathrm{A}^{\mathrm{f} / / \mathrm{ll}}$ mice leading to T-cell lymphopenia (Fig. 3b,c). Analysis of DN cells from $V a v$-creNfatcl $\alpha \mathrm{A}^{\mathrm{ff} / \mathrm{fl}}$ mice to understand the reason behind the low thymic cellularity revealed a dose-dependent block in the transition of DN3 cells to the DN4 stage (Fig. 3d; Supplementary Fig. 3a). Confirming our earlier report regarding NFATc1mediated regulation of $\mathrm{Bcl}-2$ expression in developing $\mathrm{DN}$ thymocytes, enforced NFATc1 $\alpha$ expression enhanced Bcl-2 levels in Vav-CreNfatc1 $\alpha \mathrm{A}^{\mathrm{fl} / \mathrm{fl}} \mathrm{DN} 3$ cells compared with WT 
a

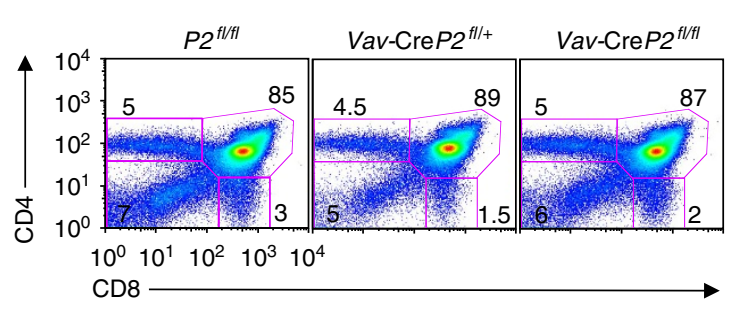

b

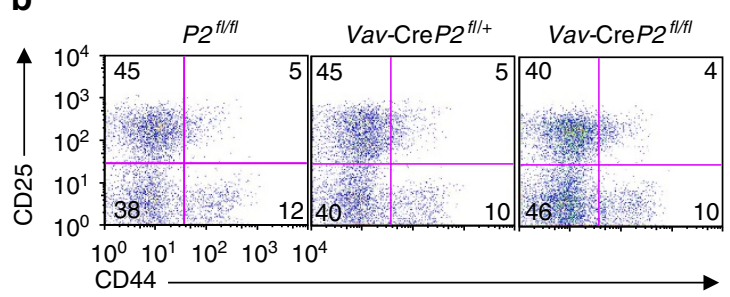

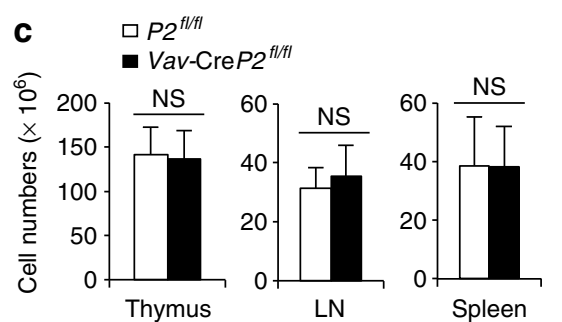

d

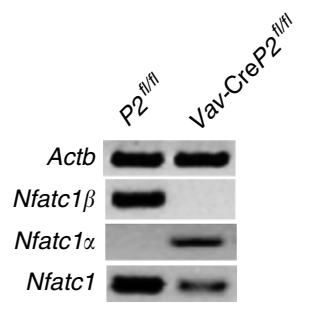

Figure 2 | Nfatc1 P1 promoter activity compensates for the loss of P2 activity in T-cell development. (a) Distribution of thymocyte subsets based on CD4 and CD8 expression in $P 2^{f l / f l}, V a v-C r e P 2^{f l}+$ and Vav-CreP2 ${ }^{f / f l}$ mice. (b) DN1-DN4 cells distribution within DN thymocytes of Vav-CreP2 $2^{f / f l}$ mice compared with that in littermate control mice based on CD44 and CD25 expression. (c) Cellularity in thymus, LNs and Spleen from P2 ${ }^{\mathrm{fl} / \mathrm{fl}}$ ( $n=10$ ) and $\operatorname{Vav}-\mathrm{CreP}^{\mathrm{fl} / \mathrm{fl}}(n=11)$ mice. (d) RT-PCR analysis for Nfatc1, Nfatcl $\alpha$ and Nfatc1 $\beta$ expression in pTCR-negative (DN1+2 +3 ) thymocytes from P2 ${ }^{f \mid / f l}$ and $\operatorname{Vav}-\mathrm{CreP} 2^{\mathrm{fl} / \mathrm{fl}}$ mice ( $n=8$ per group). Data are representative of three independent experiments and are shown as mean $\pm \mathrm{s}$.d., NS, not significant, unpaired t-test.

cells (Fig. 3e). This observation further suggests that the reduced thymic cellularity in the $V a v$-creNfatc $1 \propto \mathrm{A}^{\mathrm{fl} / \mathrm{fl}}$ mice was not because of an increase in cell death of the DN thymocytes. Similar to the $\Delta$ Cam mice ${ }^{9}$, enforced NFATc1 $\alpha$ A expression in DN3 cells resulted in the lack of rearranged TCR $\beta$ expression (Fig. 3f), leading to the loss of pTCR signalling, although the expression of CD3 $\varepsilon$ a component of the pTCR complex remained unaffected (Supplementary Fig. 3b). In addition, NFATcl $\alpha$ A activity in pTCR-negative cells strongly retarded their rate of differentiation to the double-positive (DP) and later stages. In in vitro co-culture assays on OP9-DL1 (bone marrow stromal cells expressing Notch ligand delta-like 1) monolayer, $V a v$-CreNfatcl $\alpha \mathrm{A}^{\mathrm{fl} / \mathrm{fl}} \mathrm{DN} 1-\mathrm{DN} 4$ cells showed inefficient differentiation to DP stage compared with the cells from WT mice (Fig. 3g). These observations suggest that the combined activity of NFATc $1 \alpha$ and NFATc1 $\beta$, or rather an increase in total NFATcl activity, before pTCR-positive stages is detrimental for T-cell development.

To rule out the possibility that the negative impact on thymocyte development in the $V a v$-CreNfatcl $\alpha \mathrm{A}^{\mathrm{f} / \mathrm{fl}}$ mice was due to an excess in NFATc1 activity derived from both the endogenous as well as the transgene, we generated a mouse model where only NFATc1 $\alpha$ A was expressed without any contribution from the endogenous $N f a t c 1$ gene. We bred $V a v$-CreNfatc $1 \propto \mathrm{A}^{\mathrm{fl} / \mathrm{fl}}$ mice with $N f a t c 1^{\mathrm{fl} / \mathrm{fl}}$ mice to eliminate all NFATcl activity derived from the endogenous $N f a t c 1$ gene. Analysis of $V a v-C r e N f a t c 1 \alpha A^{\mathrm{fl} / \mathrm{fl}} N f a t c 1^{\mathrm{f} / \mathrm{fl}}$ mice revealed that even in the absence of any endogenous NFATc1, NFATcl $\alpha$ activity derived from the knocked-in gene only resulted in a similar block in thymocyte differentiation at the DN3 stage compared with littermate controls (Supplementary Fig. 3c,d), as observed in the Vav-CreNfatcl $\alpha A^{\mathrm{fl} / \mathrm{fl}}$ mice. This could still be due to an above threshold level of NFATc1 activity derived from the transgene. Thus, from our analysis it is apparent that a certain threshold level of NFATc1 activity is essential for T-cell development.

NFATcl suppresses lineage plasticity of immature thymocytes. $\mathrm{T}$-cell development in the thymus follows a sequential process of
T-lineage specification, and commitment. While DN1 cells retain the potential to differentiate into B cells, natural killer (NK) cells, dendritic cells (DCs) and macrophages, the lineage plasticity gets restricted to NK and DC lineages in DN2 cells, and finally is completely lost at the DN3 stage ${ }^{13}$. Accordingly, only in WT DN1 cells we observed Ebf1 and Pax 5 expression necessary for B-cell development, whereas, expression of $I d 2$ necessary for NK cell, or Spf1 (PU.1), Csf1r and Cebpa, necessary for myeloid-lineage development were maintained in DN2 cells (Fig. 4a). We assumed that NFATc1 $\beta$ activity most likely play a role in suppressing the lineage plasticity during DN1 to DN3 differentiation and thereby consolidate T-lineage commitment. To investigate this possibility, we analysed lineage-specific gene expression in DN1-DN4 cells from $\mathrm{Vav}$-CreP2 $2^{\mathrm{fl} / \mathrm{fl}}$ mice. We observed a similar suppression of lineage plasticity towards non- $T$ lineages in the absence of NFATc1 $\beta$ activity (Fig. $4 \mathrm{~b}$ ), as in the WT mice (Fig. $4 \mathrm{a}$ ). As in the $V a v-C r e P 2^{\mathrm{fl} / \mathrm{fl}}$ mice P1 promoter-driven NFATcl $\alpha$ activity compensated for the loss of NFATc1 $\beta$ during T-cell development, we concluded that a threshold level of NFATc1 activity irrespective of any particular isoform might be crucial in suppressing lineage plasticity and thereby facilitate $\mathrm{T}$-lineage commitment.

DN1 cells are a heterogeneous population, and based on CD24 (heat-stable antigen) and CD117 (c-Kit) expression they are characterized to have five distinct populations termed as DN1a, DN1b, DN1c, DN1d and DN1e (ref. 13). DN1a and DN1b cells maintain highest lineage plasticity, whereas, DN1d and DN1e cells are more specified to take the T-cell lineage. We presumed that the combined activity of NFATcl $\alpha$, and NFATc1 $\beta$ is essential for irreversible T-lineage commitment at the pTCR-positive stage, and NFATc1 $\alpha$ if expressed along with NFATc1 $\beta$ early at the pTCR-negative stages will suppress the lineage plasticity more effectively. Interestingly, analysis of Vav-CreNfatcl $\alpha \mathrm{A}^{\mathrm{fl} / \mathrm{fl}}$ mice showed a strong reduction in DN1a and DN1b populations compared with littermate WT mice (Fig. 4c,d). Accordingly, we observed a dose-dependent specific loss of $\mathrm{B}$ cells in the thymus from Vav-CreNfatc $1 \propto \mathrm{A}^{\mathrm{fl} / \mathrm{fl}}$ mice compared to WT mice whereas, NK, DC or macrophage lineages 
a

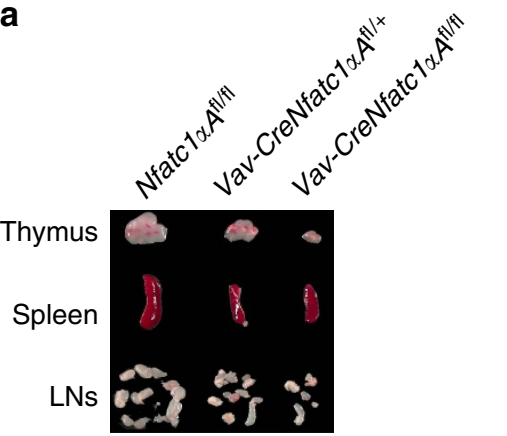

C
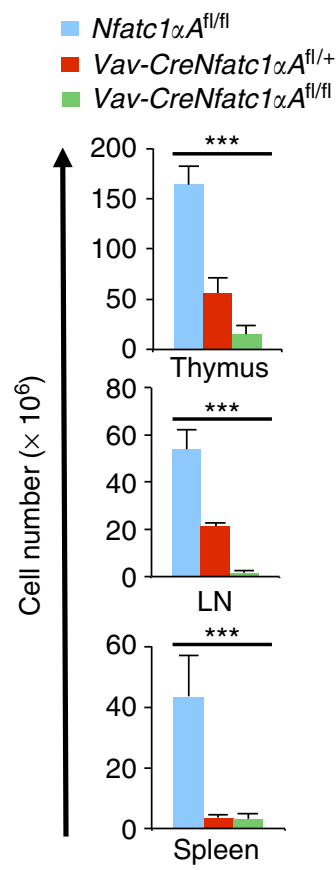

e b

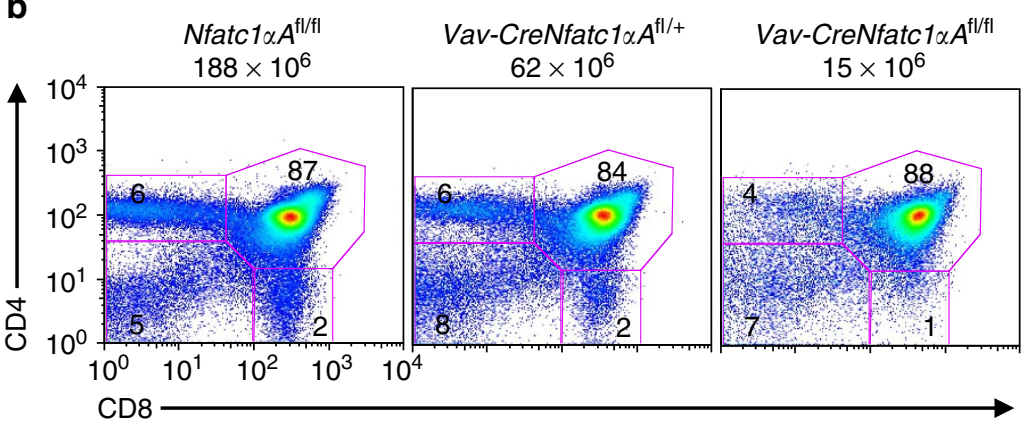

d
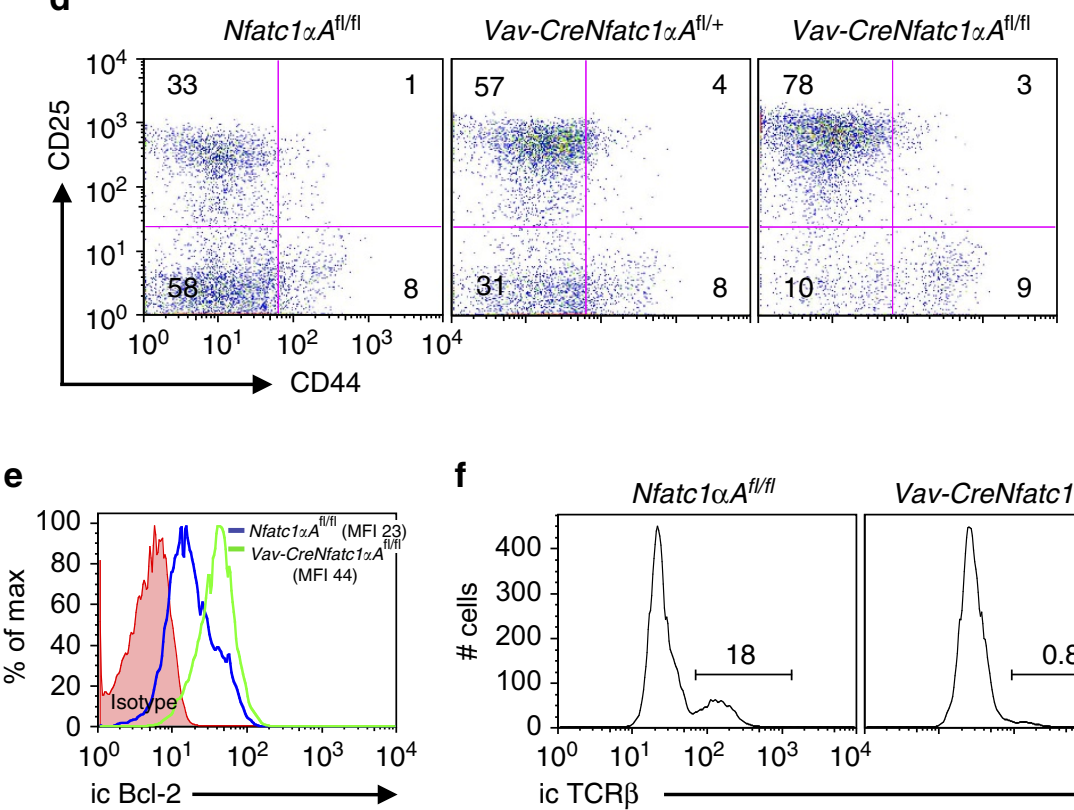

f

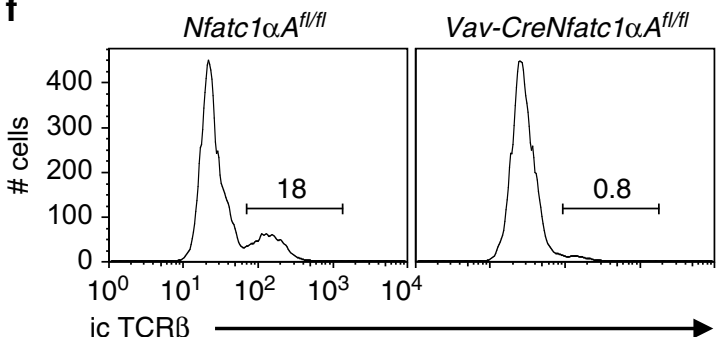

g

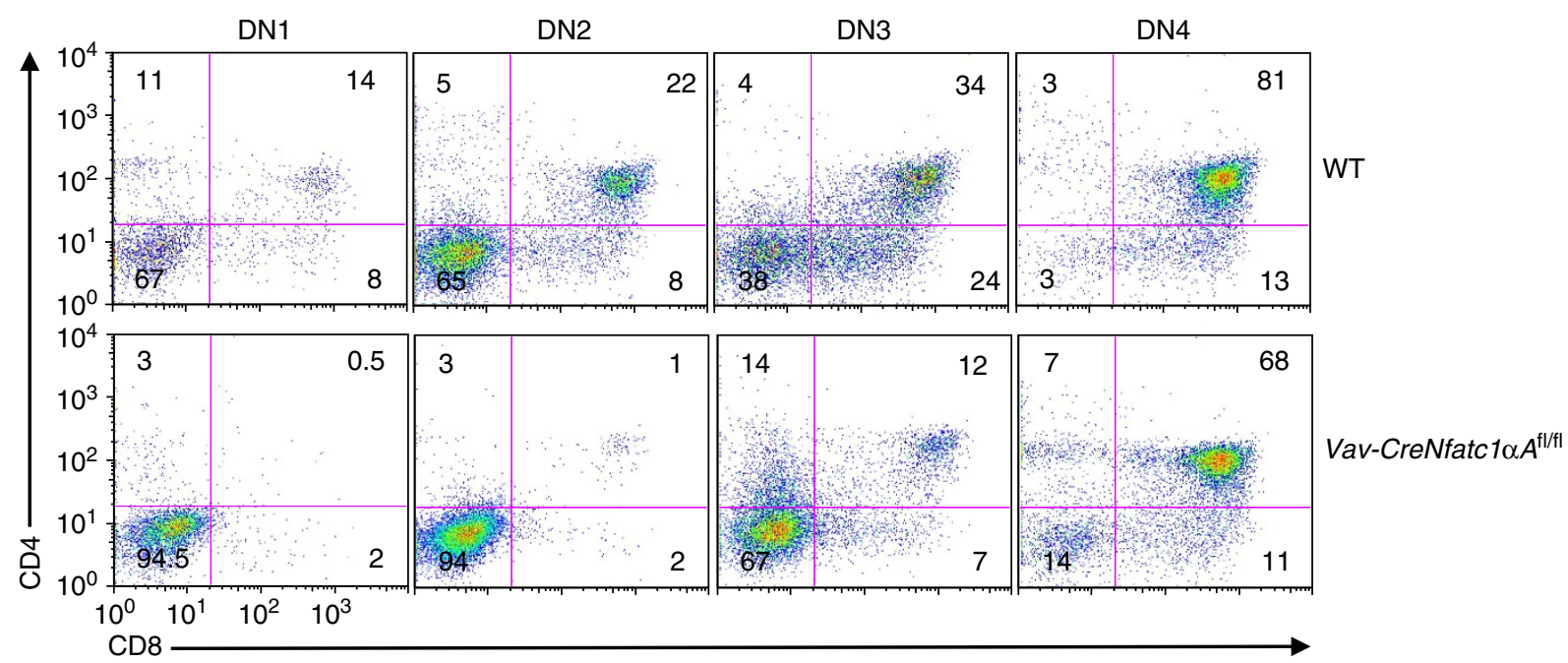

Figure 3 | Nfatc1 P1 activity in addition to P2 activity in pTCR-negative cells impairs thymocyte differentiation. (a) Photograph showing the dosagedependent effect of Nfatc1 P1 activity on the size of the thymus, LNs and spleen in Vav-CreNfatcla $\mathrm{A}^{\mathrm{fl} /}+$ and Vav-CreNfatclaA $\mathrm{fl}^{\mathrm{fl} f \mathrm{l}}$ mice compared with $N f a t c 1 \alpha A^{f l / f l}$ control mice. (b) Distribution of thymocyte subsets based on CD4 and CD8 expression in indicated mice. Number above each plot represents

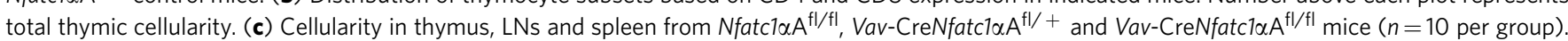
(d) Distribution of DN1-DN4 cells based on CD44 and CD25 expression among DN thymocytes from indicated mice. (e) Intracellular Bcl-2 expression in Vav-CreNfatc1 $\alpha \mathrm{A}^{\mathrm{fl} / \mathrm{fl}} \mathrm{DN3}$ cells compared with that in Nfatc1 $\alpha \mathrm{A}^{\mathrm{fl} / \mathrm{fl}}$ control mice. MFI, mean fluorescence index. (f) Intracellular TCR $\beta$ expression in DN3 cells from indicated mice. Number inside each plot represents per cent TCRß-positive cells. (g) Impaired differentiation of Vav-CreNfatciaA ${ }^{\mathrm{fl} / f l}$ DN1-DN4 cells to DP stage on OP9-DL1 monolayer compared with WT cells in vitro. Numbers inside each plot represent per cent respective population. Data represent one of three independent experiments ( $n=4$ per group), and are shown as mean \pm s.d., ${ }^{\star \star \star} P<0.0001$, one-way analysis of variance. 


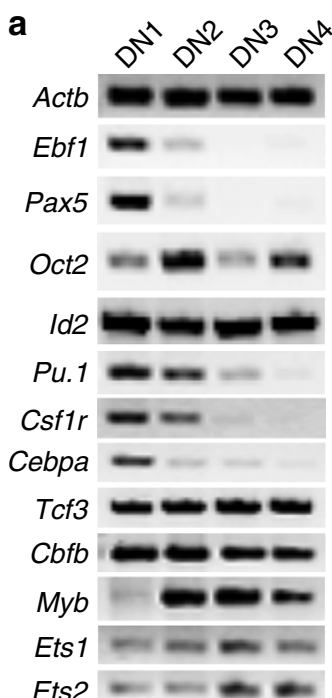

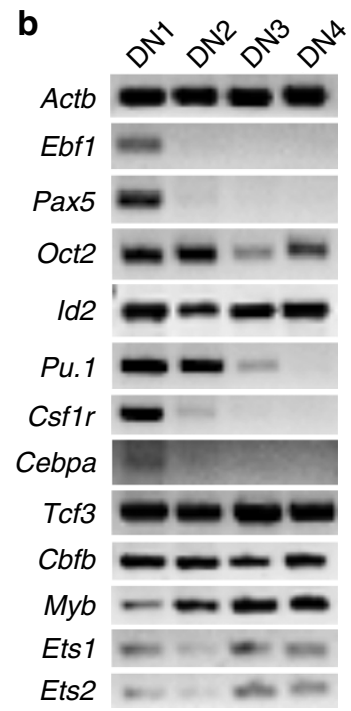

C

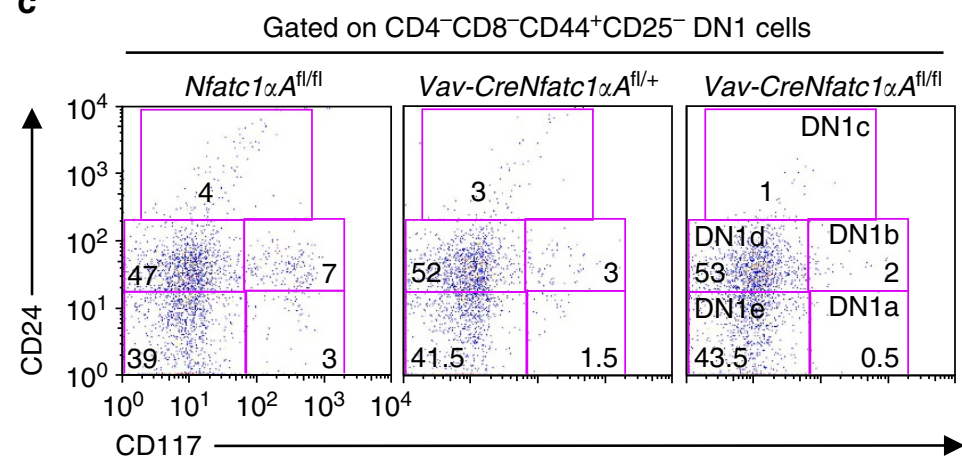

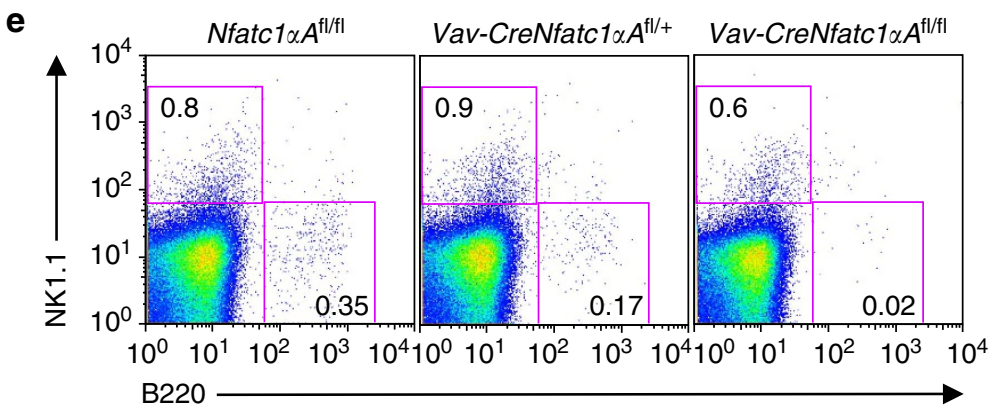

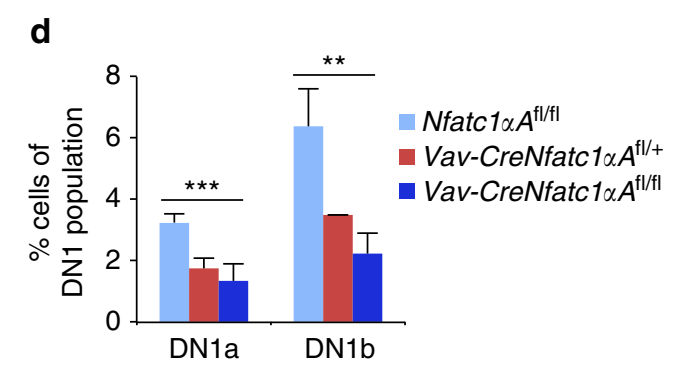

f

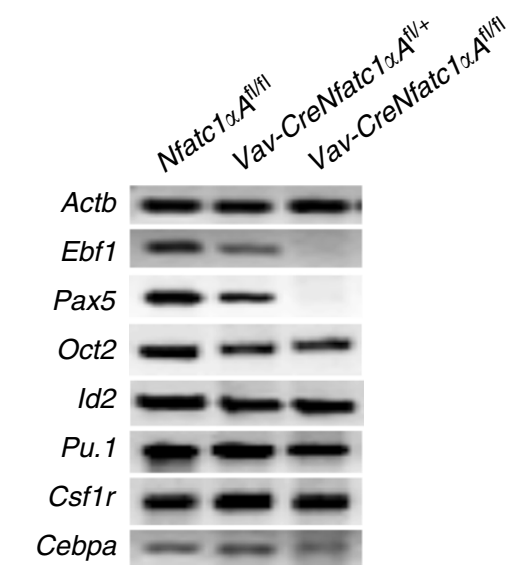

Figure 4 | NFATc1 activity suppresses B-lineage potential of immature thymocytes. (a) RT-PCR analysis of B, NK, macrophage and DC lineage specifying genes expression in DN1-DN4 cells from WT mice. (b) Gene expression analysis on cells as mentioned in a from Vav-CreNfatc1P2 ${ }^{f l / f l}$ mice. (c) Analysis of

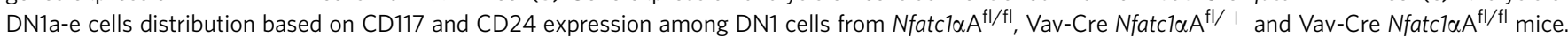
(d) Distribution of DN1a and DN1b cells within DN1 population in Vav-CreNfatcl $\alpha A^{\mathrm{fl} / \mathrm{fl}}(n=6)$ and Vav-CreNfatcl $\alpha \mathrm{A}^{\mathrm{fl} /}+(n=6)$ mice compared to control $N f a t c 7 \alpha A^{f l / f l}(n=4)$ mice. (e) Flow cytometry profiles reveal the distribution of $B$, NK, macrophage and DC populations in the thymus of indicated mice. (f) RT-PCR analysis of B, NK, macrophage and DC lineage specifying genes expression in DN1 cells from Vav-Cre Nfatcl $1 \alpha \mathrm{A}^{\mathrm{fl} / \mathrm{fl}}$ mice compared with Vav-Cre $N f a t c 1 \alpha A^{f l /}+$ and $N f a t c 1 \alpha A^{f l / f l}$ control mice. Numbers inside each plot represent per cent respective population. Data are representative of three independent experiments and are shown as mean \pm s.d., ${ }^{\star \star \star} P=0.0007$ and ${ }^{\star \star} P=0.0013$, one-way analysis of variance.

were not affected (Fig. 4e). Corroborating this, Ebf1 and Pax5 expression were suppressed in Vav-CreNfatcl $\alpha \mathrm{A}^{\mathrm{fl} / \mathrm{fl}} \mathrm{DN1}$ cells, whereas gene expression necessary for NK and myeloid lineages was unaffected (Fig. 4f). However, the suppression of B-lineage potential of the Vav-CreNfatcl $\alpha \mathrm{A}^{\mathrm{fl} / \mathrm{fl}} \mathrm{DN} 1$ cells was neither due to enhanced cell death nor due to any toxic effect of NFATc1 activity on the precursor cells. In a B-lineage permissible environment, FACS sorted DN1 thymocytes from Vav-CreNfat$c 1 \alpha A^{\mathrm{fl} / \mathrm{fl}}$ mice developed comparable proportion of $\mathrm{B} 220^{+} \mathrm{B}$ cells as that in case of WT DN1 cells, when co-cultured on OP9 bone marrow stromal cell layer (Supplementary Fig. 3e). This observation ruled out that there was any inherent developmental restriction towards B-lineage differentiation in the Vav-CreNfat$c 1 \propto \mathrm{A}^{\mathrm{fl} / \mathrm{fl}} \mathrm{DN} 1$ cells.

Integrin signalling induces $N$ fatc1 $\mathrm{P} 2$ promoter activity. The exclusive P2 activity in the WT pTCR-negative thymocytes led us to ask how the P2 promoter is regulated in these cells. Surprisingly, when we cultured DN4 thymocytes in vitro, not only all P1-directed transcripts disappeared but we also observed the absence of all P2-derived transcripts as well (Fig. 5a). However, annexin $\mathrm{V}$ analysis revealed that majority of cells in this culture condition were alive, ruling out cell death being the reason for the 


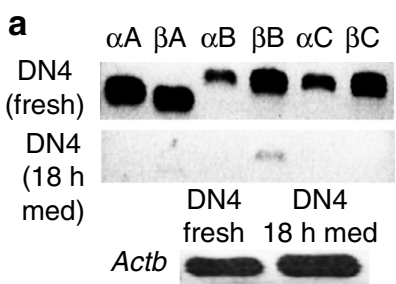

b $\quad \alpha A \beta A \alpha B B \quad \alpha C$

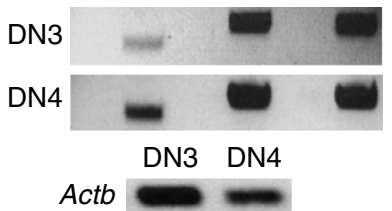

e

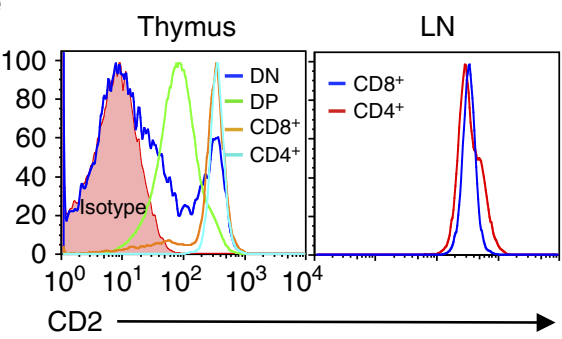

C

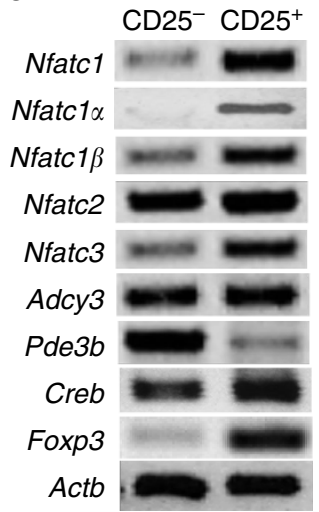

d
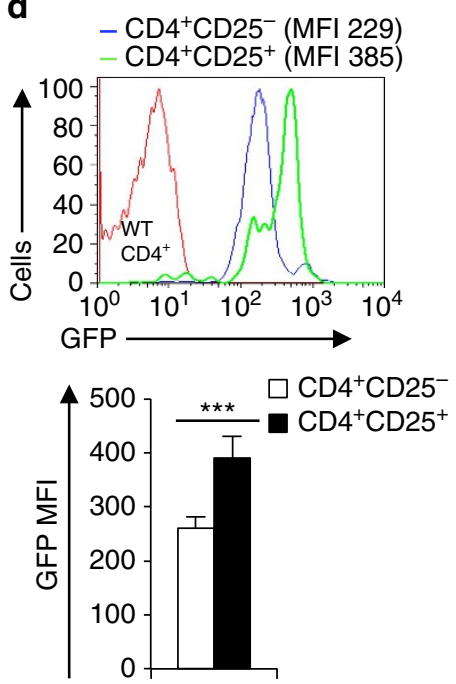

f

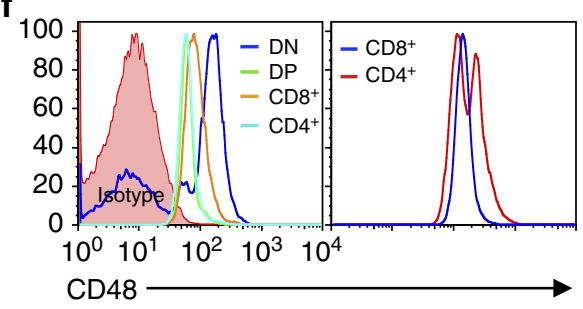

g

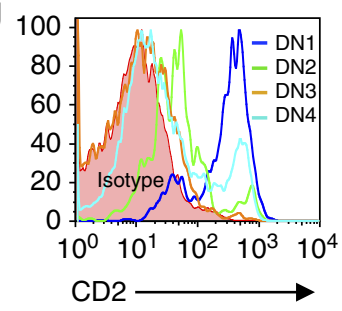

DN3 DN4 DP CD4 ${ }^{+}$

h

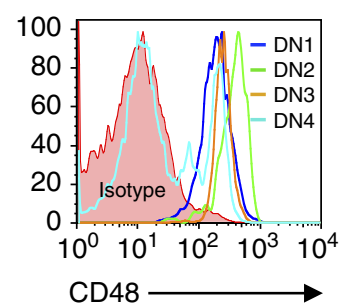

$\mathbf{k}$

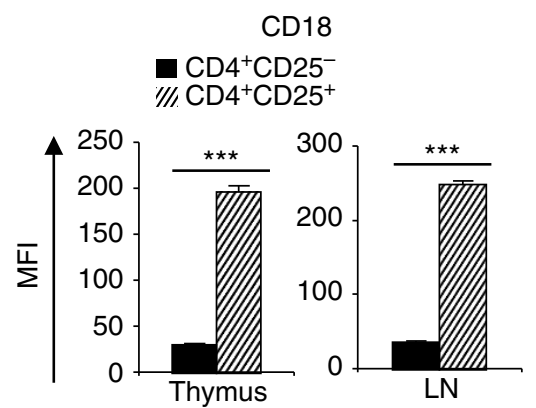

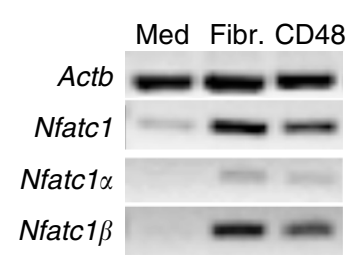

j

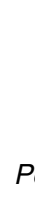

Itga6

Itgav (CD51)

Pecam1 (CD31)

Itgb2 (CD18)

Itga4 (CD49d)

I
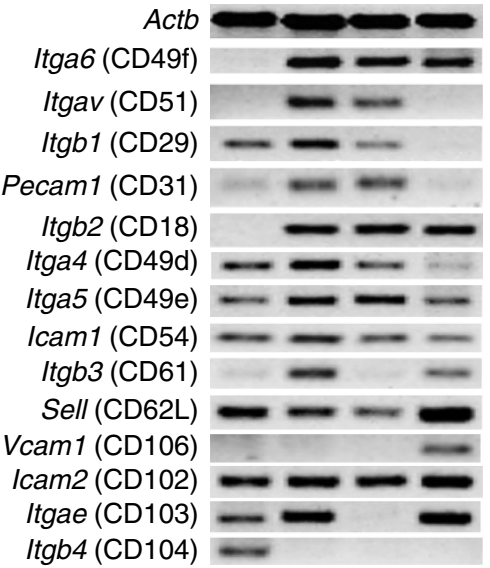
Foxp3- effector $\mathrm{T}\left(\mathrm{T}_{\text {eff }}\right)$ cells (Fig. 5c). This observation was corroborated with higher level of green fluorescent protein (GFP) expression reflecting higher NFATc1 levels in $\mathrm{T}_{\text {reg }}$ cells against $\mathrm{T}_{\text {eff }}$ cells from Nfatc1-eGfp-Bac tg reporter mice ${ }^{17}$ (Fig. $5 \mathrm{~d}$ ).

Next, we investigated which signalling pathway is involved in generating intracellular cAMP that drives Nfatcl expression in thymocytes and in $\mathrm{T}$ cells. Cell-cell interaction is mediated by various integrins, and thymocytes, as well as $\mathrm{T}$ cells express a number of integrins. CD2 (LFA-2) expressed on thymocytes and $\mathrm{T}$ cells has been shown to activate $\mathrm{T}$ cells ${ }^{18,19}$. Murine CD2 binds to its ligand CD48 expressed on $\mathrm{T}$ cells as well as on the interacting cells and thereby can transduce signals both in cis and in trans ${ }^{20-23}$. In addition, CD2 signalling in $\mathrm{T}$ cells have been reported to induce $\mathrm{CAMP}^{24,25}$, and thereby the activation of cAMP response element binding (CREB) proteins ${ }^{26}$. Mice lacking CAMP-CREB signalling show impaired thymocyte proliferation and altered fetal T-cell development ${ }^{27,28}$. To check whether CD2-
CD48 interactions regulate $\mathrm{Nfatcl}$ expression in DN thymocytes, we analysed CD2 and CD48 expression on various thymocyte subsets. We observed only a fraction of DN thymocytes expressed CD2 on their surface, which was increased in DP and SP thymocytes as well as in peripheral $\mathrm{T}$ cells (Fig. 5e). However, CD48 expression was highest on DN cells and lowest in DP and $\mathrm{CD}^{+}{ }^{+} \mathrm{SP}$ cells (Fig. 5f). Among DN thymocytes, DN1 cells expressed the highest level of CD2, whereas CD48 expression was high on all pTCR-negative DN populations (Fig. 5g,h). To prove whether CD2-CD48 signals could induce $N f a t c 1$ expression, we stimulated WT DN3 cells with CD48 antibodies and analysed for the generation of $\mathrm{P} 1-$ and P2-directed transcripts. CD48 stimulation at a higher concentration induced the synthesis of both P1- and P2-directed Nfatc1 transcripts in DN3 cells, while stimulation with PMA, reported to substitute CD2 co-stimulation $^{29}$, did not generate any $N$ fatc1 RNA (Fig. 5i). However, CD48 at low concentration only induced Nfatc1 P2
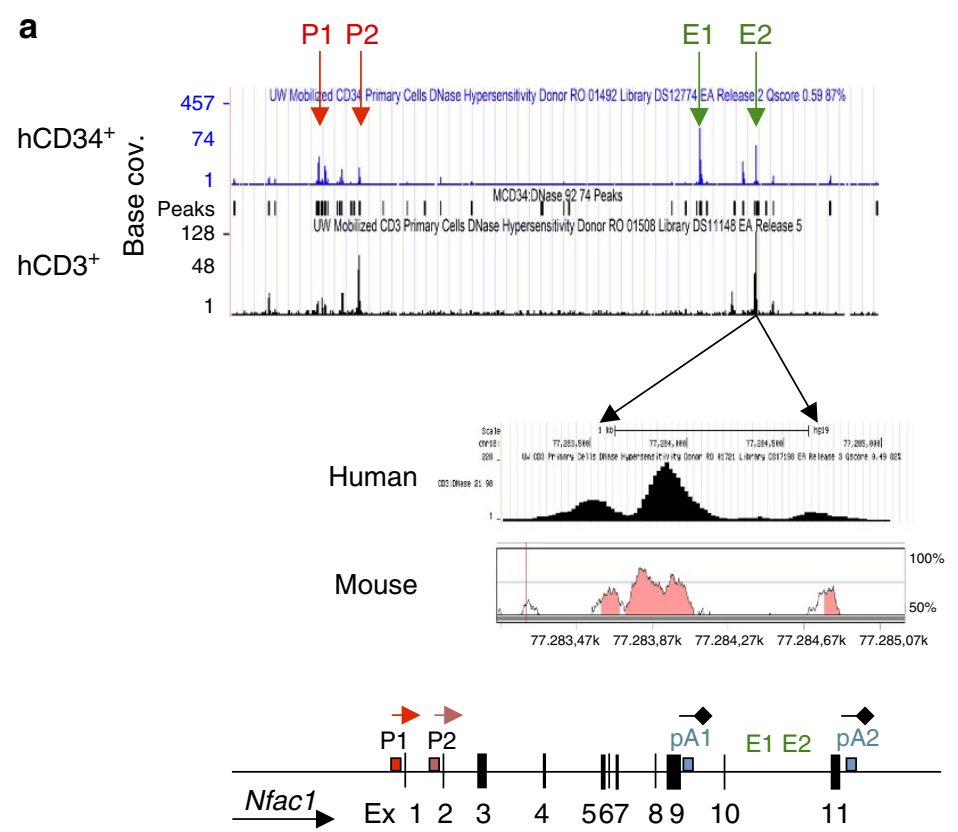

d

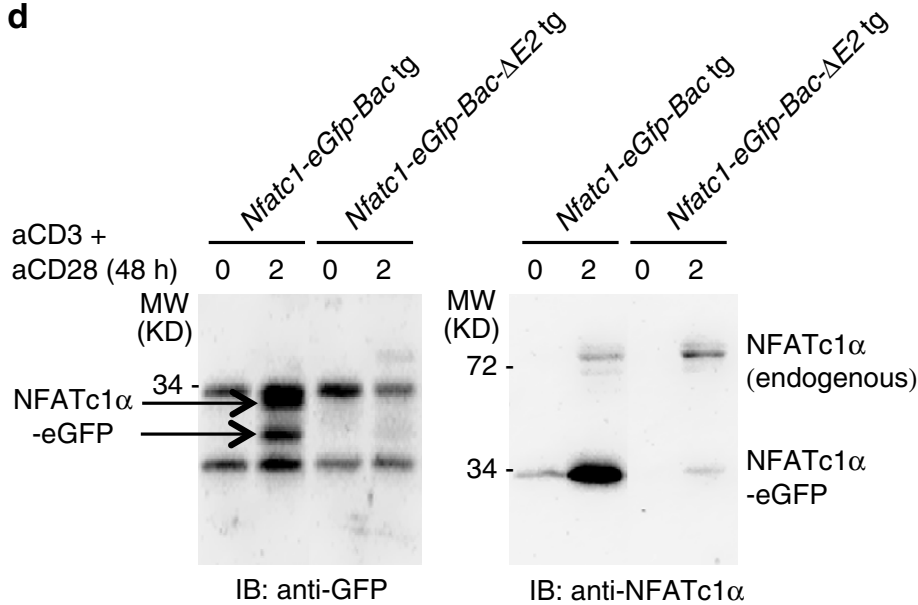

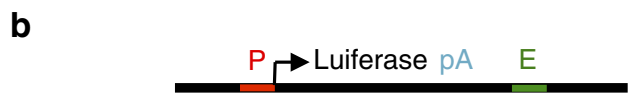

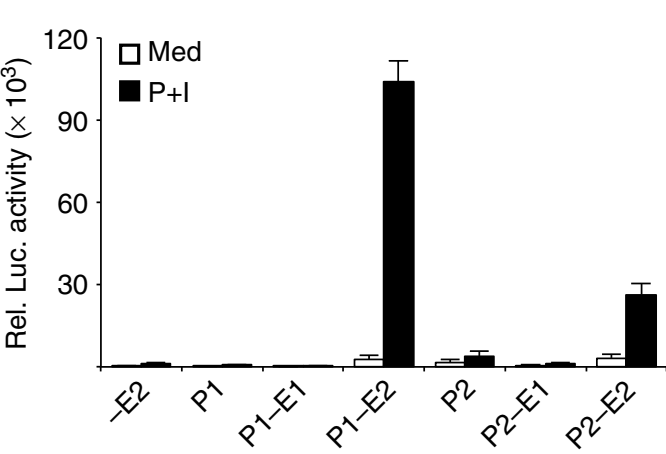

c

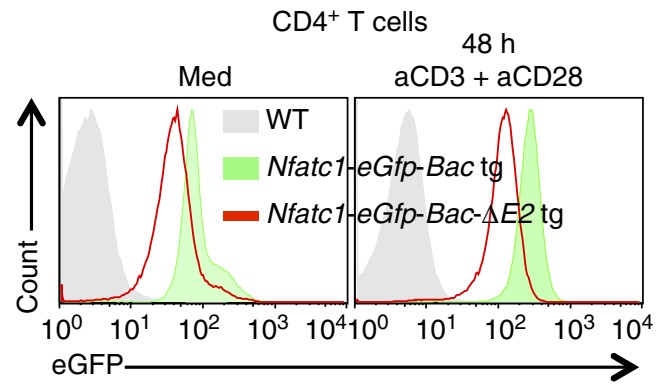

Figure 6 | Nfatc1 P1 activity is induced by a novel enhancer element. (a) DNase I hypersensitive sites in the NFATc1 locus in human hematopoietic stem and $\mathrm{CD}^{+}{ }^{+}$T cells. Their corresponding position in the murine Nfatc1 gene is also shown. (b) Enhancer activity of the E2 element in inducing Nfatc1 P1 promoter activity in reporter assays. (c) NFATc1 expression levels as revealed by GFP expression in freshly isolated, or aCD3 + aCD28 Abs stimulated $\mathrm{CD}^{+}{ }^{+}$T cells from Nfatc1-eGfp-Bac- $\Delta E 2$ tg mice compared with that from Nfatc1-eGfp-Bac tg mice. (d) Immunoblot analysis for GFP or NFATc1 $\alpha$ expression in unstimulated and $48 \mathrm{~h}$ aCD3 + aCD28 Abs stimulated CD4 ${ }^{+}$T cells from Nfatc1-eGfp-Bac- $\Delta E 2$ tg mice compared with that from Nfatc1eGfp-Bac tg mice. 
activity in the pTCR-negative thymocytes unraveling the effect of signal strength on the inducibility of P1 and P2 promoters (Supplementary Fig. 4b).

Although CD2-CD48 signalling induced Nfatc1 expression in vitro, these are unlikely to be the only integrins responsible for Nfatcl expression in vivo. This was supported by the reported normal T-cell development in $C d 2^{-1-}$ mice $^{30-32}$, as well as in mice injected with anti-CD2 antibodies ${ }^{33}$, and also in $C d 48^{-1-}$ mice ${ }^{34}$, indicating the involvement of additional integrins in regulating Nfatcl expression. Accordingly, analysis of thymocytes and $\mathrm{T}$ cells showed a differential expression pattern for various integrins (Supplementary Fig. 4c; Fig. 5j). $\mathrm{CD} 4{ }^{+} \mathrm{CD} 25^{+} \mathrm{T}_{\text {reg }}$ cells both in the thymus and in LNs expressed much higher levels of various integrins compared to $\mathrm{CD} 4{ }^{+} \mathrm{CD} 25^{-} \mathrm{T}_{\text {eff }}$ cells (Fig. 5k; Supplementary Fig. 4d), concurring with our observation regarding higher Nfatcl expression in $\mathrm{T}_{\text {reg }}$ cells over $\mathrm{T}_{\text {eff }}$ cells (Fig. 5c). Among the $\mathrm{CD} 4^{+} \mathrm{CD} 25^{+}$population, $\mathrm{CD} 25^{\mathrm{hi}}$ cells expressed highest level of various integrins, the most prominent being CD18, CD48, CD49d and CD62L (Supplementary Fig. 5a,b). This integrin expression pattern directly correlated to that of $\mathrm{Nfatc1}$ expression in $\mathrm{CD} 25^{\text {hi }}$ cells as revealed from higher GFP levels in the Nfatc1-eGfp-Bac transgenic reporter mice (Supplementary Fig. 5c). To further consolidate our observation, we stimulated $\mathrm{WT} \mathrm{CD} 4{ }^{+} \mathrm{T}$ cells with fibronectin, the ligand for CD18. Interestingly, fibronectin stimulation induced robust Nfatcl expression, which was mainly derived from the P2 promoter (Fig. 5l). In addition, similar to that in DN3 thymocytes (Fig. 5i), CD2-CD48 signalling also induced $\mathrm{Nfatc1} \mathrm{P2} \mathrm{activity} \mathrm{in}$ peripheral $\mathrm{CD}^{+}{ }^{+} \mathrm{T}$ cells (Fig. 5l). Thus, the above observations established integrin-cAMP signalling as a critical component in regulating $\mathrm{Nfatcl}$ gene expression in thymocytes and in $\mathrm{T}$ cells.

An intronic cis-regulatory element controls P1 activity. To address the question why $\mathrm{P} 1$ promoter is inactive in the pTCR-negative thymocytes, we analysed DNA methylation status at the $\mathrm{P} 1$ promoter in these cells. However, methylation analysis did not reveal any significant difference between WT DN3 and DN4 cells (Supplementary Fig. 6a). To investigate whether epigenetic modifications on pTCR signalling at the DN3 to DN4 transition induce P1 activity we performed ChIP-Seq analysis of Rag2 ${ }^{-I}$ DN3 cells stimulated with anti-CD3 Abs to mimic pTCR signals. However, no additional epigenetic changes were detected at the P1 promoter in the anti-CD3 stimulated Rag2 ${ }^{-1}$ DN3 cells compared to the unstimulated cells (Supplementary Fig. 6b; Fig. 1b). These observations suggested that P1 promoter induction still requires involvement of additional cis-regulatory elements and/or trans-acting factors. To identify cis-regulatory elements essential for P1 promoter activity, we studied the DNase I hypersensitivity analysis of the human NFATc1 locus (Roadmap Epigenomics Project, www.roadmapepigenomics.org). Besides the P1 and P2 promoter elements, two very distinct DNase I hypersensitive sites designated as E1 and E2 were evident in the intron between exons 10 and 11 in the $\mathrm{CD} 4^{+}$human haematopoietic stem cells (Fig. 6a). Significantly, these elements were tissue-specific as compared with the hematopoietic stem cells, $\mathrm{CD}^{+}$human $\mathrm{T}$ cells showed only the presence of $\mathrm{E} 2$ site, which was conserved in murine $\mathrm{T}$ cells as well (Fig. 6a).

To investigate the influence of E1 and E2 elements on Nfatc1 expression, we generated luciferase reporter constructs with the $\mathrm{P} 1$ or $\mathrm{P} 2$ promoter in combination with the $\mathrm{E} 1$ or $\mathrm{E} 2$ element (Fig. 6b). On stimulation with PMA + Ionomycin (I), in EL-4 thymoma cells the E2, but not the E1 element induced a strong Nfatc1 P1 promoter activity compared with a mild increase in $\mathrm{P} 2$ promoter activity (Fig. 6b). We also tested two additional DNAse
I hypersensitive sites, E3 positioned in the intron between exons 3 and 4 , and E4 positioned at the extreme $3^{\prime}$ region of $N f a t c 1$ locus for their potential regulatory effect on $\mathrm{Nfatcl}$ promoter activity. However, both these elements were ineffective in inducing $\mathrm{Nfatcl}$ P1 promoter activity (Supplementary Fig. 6c), suggesting the essentiality of the E2 element in the context of P1 promoter activity.

To extend our characterization of the E2 enhancer, we analysed for the trans-acting factors, which might play a role in enhancing the E2-mediated P1 promoter activity. We detected binding motifs for three prominent lymphoid-specific transcription factors; NFATc1, GATA3 and PU.1 in the E2 element (Supplementary Fig. 6d). To investigate whether any of these factors positively modulates the E2 enhancer activity, we mutated the binding sites for individual factors and checked their enhancer potential for Nfatcl P1 promoter activity. In reporter assays, only the NFATc1 mutant showed loss of enhancer activity, whereas GATA3 or PU.1 mutants exerted no influence on Nfatc1 P1 promoter activity (Supplementary Fig. 6d). This was in line with the previous reports from our laboratory that NFATc1 autoregulates the P1 promoter activity ${ }^{5}$, however, the involvement of the E2 element was hitherto unknown.

Further, to study the effect of E2 element on $\mathrm{Nfatc1}$ promoter in vivo, we generated Nfatcl-eGfp-Bac- $\Delta E 2$ tg reporter mice by deleting $1 \mathrm{~Kb}$ DNA from intron 10 harbouring the E2 element (Supplementary Fig. 6e $)^{17}$. Loss of E2 enhancer activity in Nfatc1eGfp-Bac- $\triangle E 2$ tg reporter mice, showed a clear reduction in Nfatcl expression in $\mathrm{CD} 4^{+} \mathrm{T}$ cells as evident from reduced GFP expression both in unstimulated as well as in stimulated cells compared with that in the Nfatc1-eGfp-Bac tg reporter mice (Fig. 6c). This reduction in NFATc1 levels was due to a specific loss of P1-derived NFATc1 $\alpha$, as we observed a strong decrease in NFATcl $\alpha$ proteins in immunoblot analysis with antibodies against GFP as well as NFATc1 $\alpha$, whereas loss of E2 enhancer activity did not influence NFATc1 $\beta$ protein levels (Fig. 6d; Supplementary Fig. 7). Thus, we have identified a novel cis-regulatory element E2, acting as an enhancer, specifically for the P1 promoter activity in $\mathrm{T}$ cells.

NFATc1 activity prevents experimentally induced T-ALL. At the DN3 stage, though pTCR signalling is vital for proliferation and differentiation of thymocytes, it is equally crucial that the pTCR signals are switched off in the differentiated cells. Failure to do so will lead to the development of T-ALL, an aggressive form of leukaemia observed in both mouse and humans with constitutive Notch signalling ${ }^{35-40}$.

We have previously shown that T-cell development in $\Delta$ Cam mice is severely blocked at DN3 stage due to a defect in pTCR formation ${ }^{9}$. Further analysis revealed a strong downregulation in Ptcra (pT $\alpha)$ expression (Fig. 7a), in $\Delta$ Cam DN3 cells. Ptcra expression is regulated by Notch signalling ${ }^{41}$, and accordingly, in $\mathrm{N} 3$ tg T cells pT $\alpha$ expression is not extinguished ${ }^{38}$. The negative effect of NFATc1 $\alpha$ on Ptcra expression in $\triangle$ Cam DN3 cells suggests that Nfatc1 P1 activity might be involved in downregulating $\mathrm{pT} \alpha$ expression once the cells have received pTCR signals. Inhibition of NFATc1 activity by CsA treatment restored Ptcra expression in $\triangle$ Cam DN3 cells (Fig. 7b), further strengthening the possibility that NFATc1 $\alpha$ suppresses Ptcra expression. This was confirmed by a dose-dependent suppression of Ptcra expression in DN4 cells in Vav-CreNfatcl $\alpha A^{\mathrm{f} / /+}$ and Vav-CreNfatc1 $\alpha A^{\mathrm{fl} / \mathrm{fl}}$ mice compared with WT cells (Fig. 7c). Further, ChIP assays confirmed NFATcl $\alpha$ binding at the Ptcra promoter in vivo in WT DN and $\Delta$ Cam DN3 cells (Fig. 7d). Also, in reporter assays NFATc1 $\alpha$ failed to induce Ptcra promoter activity whereas, it efficiently activated the murine $I l 2$ promoter 
a

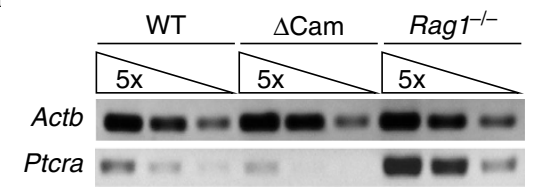

b

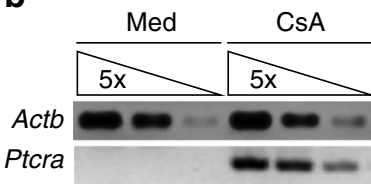

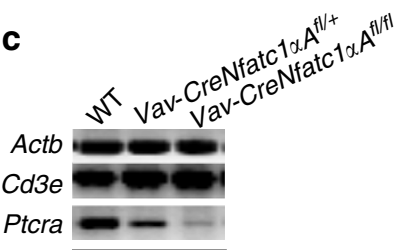

DN4 d

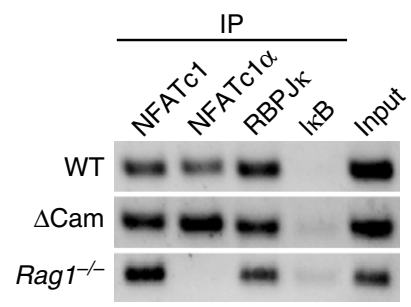

e

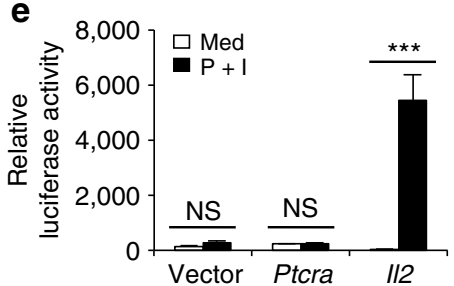

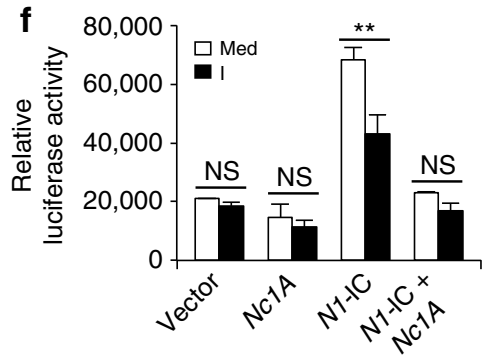

g

Gated on $\mathrm{CD}^{-}{ }^{-} \mathrm{CD} 8^{-}$cells
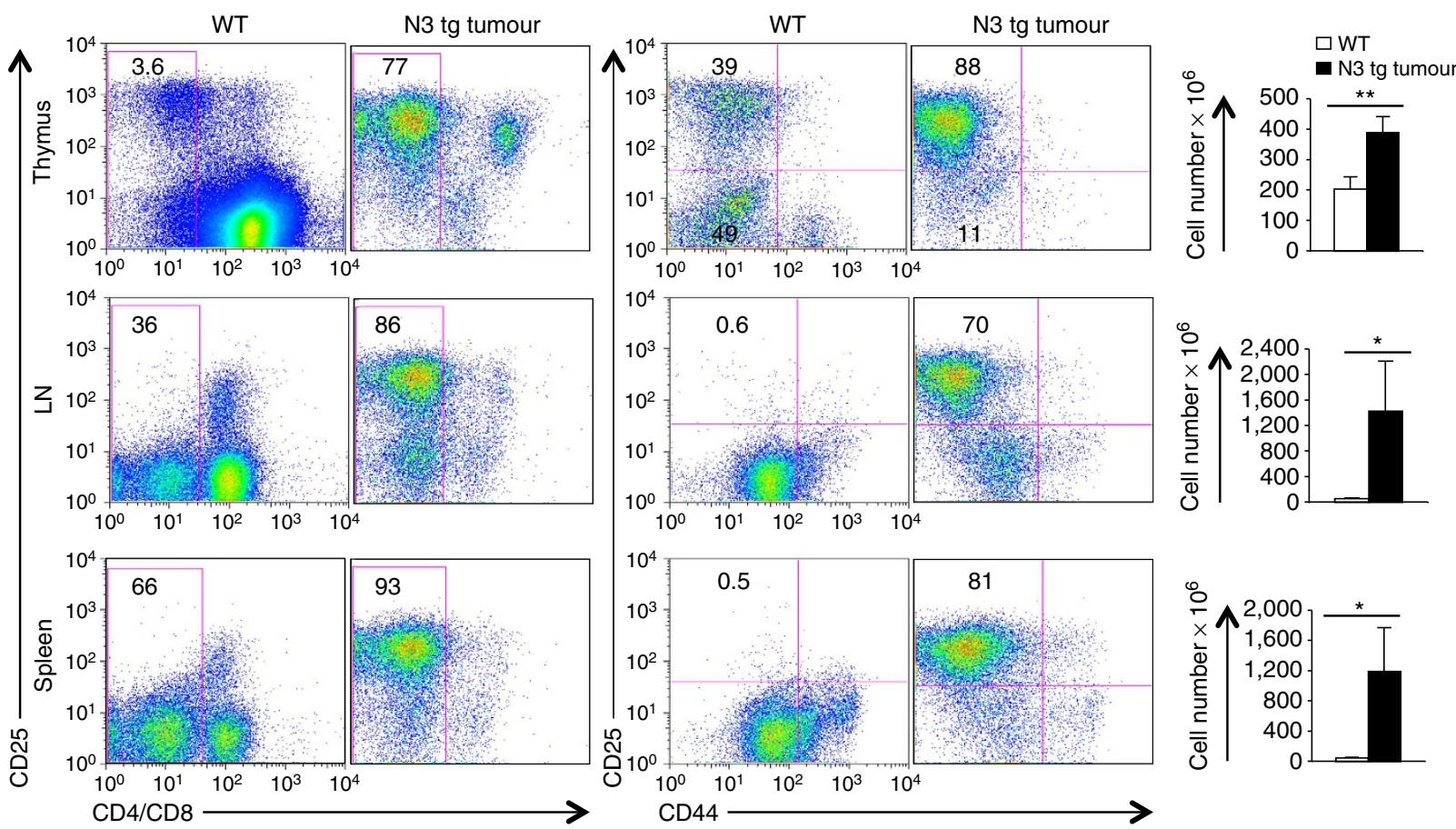

h
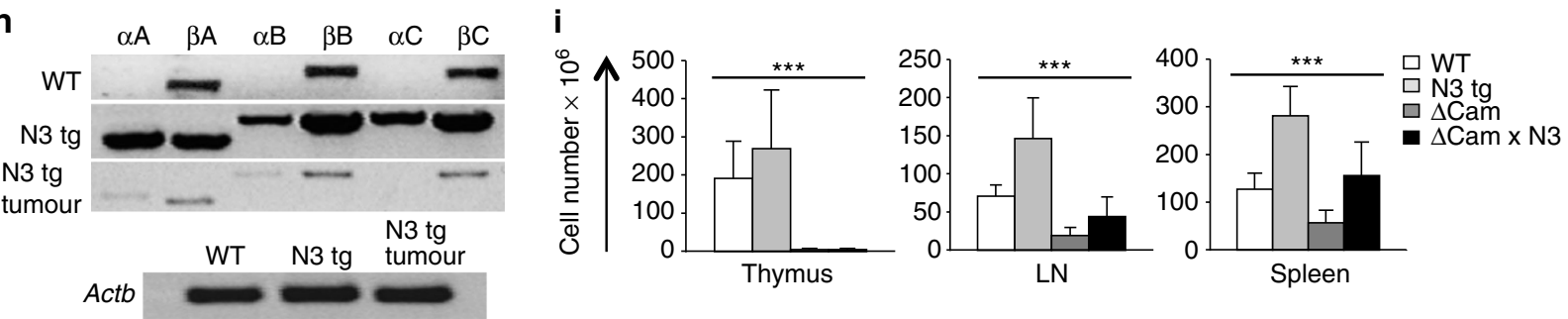

Figure 7 | NFATc1 $\alpha$ activity prevents T-ALL development. (a) RT-PCR analysis for Ptcra expression in DN3 cells from WT, $\Delta$ Cam and Rag ${ }^{-/}$- mice. (b) Ptcra expression levels in CsA treated or untreated DN3 cells from $\triangle$ Cam mice. (c) RT-PCR analysis for Cd3e and Ptcra expression in DN4 cells from

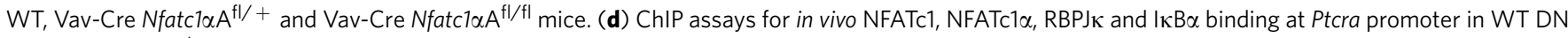
cells or in Rag1 $1^{-/}$and $\Delta$ Cam DN3 cells. (e) Luciferase reporter assay depicting the influence of NFATc1 $\alpha$ on Ptcra and II2 promoter activity in unstimulated or $\mathrm{P}+\mathrm{I}$ stimulated EL-4 thymoma cells. (f) Effects of NFATc1 $\alpha$ activity on Notch-induced Ptcra promoter transactivation in unstimulated or ionomycine (I) stimulated Jurkat T-ALL cells as revealed by luciferase reporter assays. (g) Flow cytometry profiles depicting the distribution of CD4 ${ }^{+} /$ $\mathrm{CD}^{+}$T-cell population (left panel), and $\mathrm{CD} 4^{-} \mathrm{CD} 8{ }^{-} \mathrm{CD} 44^{-} \mathrm{CD} 25^{+}$DN3 cells (right panel) in the thymus, LNs and spleen from mice with Notch3induced T-ALL compared with WT littermate controls ( $n=7$ per group). Histograms depict the cellularity in the thymus, LNs and in the spleen. (h) RT-PCR analysis of Nfatc1 isoforms expression in N3-induced T-ALL cells compared with DN3 cells from WTor normal N3 tg mice. (i) Cellularity in the thymus, LNs and Spleen from WT, N3 tg, $\Delta$ Cam and $\Delta$ Cam $\times$ N3 double-tg mice ( $n=6$ per group). Data are representative of three independent experiments and are shown as mean \pm s.d., ${ }^{\star \star \star} P<0.0001$, one-way analysis of variance. ${ }^{\star \star} P=0.0039$ or 0.0049 and ${ }^{\star} P=0.0317$ or 0.0207 , unpaired $t$-test. 
(Fig. 7e). However, in human T-ALL cell line Jurkat, in reporter assays we observed a strong downregulation in Notch-induced Ptcra promoter activity by NFATcl $\alpha$ (Fig. 7f). Thus, our observations suggest that Nfatc1 P1 activity critically influences Notch-pTCR signalling, necessary for normal T-cell development.

If this is the case, modulation of Nfatc1 P1 activity might help to prevent T-ALL pathogenesis. We used N3-induced T-ALL as a model to check the role of NFATc1 in T-ALL development. N3 tg mice developed severe T-ALL with $\mathrm{CD} 4{ }^{-} \mathrm{CD} 8{ }^{-} \mathrm{CD} 44^{-} \mathrm{CD} 25^{+}$ DN3 cells accumulating in the thymus and peripheral lymphoid organs (Fig. $7 \mathrm{~g}$ ). As a result of tumorigenic transformation the differentiation of DN3 cells to T cells was drastically blocked in $\mathrm{N} 3$ tg tumour mice (Fig. $7 \mathrm{~g}$ ). Interestingly, $\mathrm{Nfatc1}$ expression in these N3-induced T-ALL cells was strongly downregulated compared with WT DN3 cells (Fig. 7h). We hypothesized that if the suppressed Nfatcl expression is the 'cause' and not the 'effect' of T-ALL, then restoring NFATc1 activity in N3 tg mice will help prevent leukemogenesis. To check this possibility in vivo, we crossed $\Delta$ Cam mice with $\mathrm{N} 3$ tg mice to generate $\Delta$ Cam $\times$ N3 double-tg mice. Surprisingly, in contrast to N3 tg mice, none of the $\Delta \mathrm{Cam} \times \mathrm{N} 3$ double-tg mice analysed, showed any sign of T-ALL (Fig. 7i). Rather, the double-tg mice showed a thymic phenotype similar to that observed in $\Delta$ Cam mice (Fig. $7 \mathrm{i}$; Supplementary Fig. 8a). Importantly, in $\Delta$ Cam $\times$ N3 double-tg mice even though thymocytes were accumulated at the DN3 stage (Supplementary Fig. 8b), still they were not tumorigenic suggesting a strong tumour-preventing activity of NFATc1 in this experimental model.

\section{Discussion}

We have shown previously the indispensability of NFATc1 activity in early thymocyte development ${ }^{2}$. Here we have delineated the signals that control Nfatcl expression initiated from two distinct promoters in a thymocyte developmental stagespecific manner and, how NFATc1 activity simultaneously facilitates the T-cell fate of the thymocytes, and prevents the pathogenesis of T-ALL. The finding that NFATc1 expression directed from only $\mathrm{P} 2$ promoter at the pTCR-negative stages and from both $\mathrm{P} 1$ and $\mathrm{P} 2$ promoters at the pTCR-positive stages establish the differential threshold of NFATc1 activity required for thymocyte differentiation. Once the DN3 cells receive pTCR signals, they are irreversibly committed towards T-lineage only. Hence, the NFATc $1 \alpha$ activity at the pTCR-positive stages is the key to enhance the threshold of total NFATc1 activity through which T-lineage commitment is established on pTCR signals. Thus, the switch from 'NFATc1 $\beta$ only' to both 'NFATc1 $\alpha$ and $\beta$ ' at the pTCR-positive DN3 cells is absolutely necessary for T-lineage commitment in the thymus. In this regard our observation that NFATc1 activity specifically suppresses the B-lineage plasticity of the immature thymocytes provides an important clue as to how activities of various transcription factors stabilize T-cell fate during development. Importantly, the characterization of a novel enhancer element specifically regulating the $\mathrm{P} 1$ promoter activities holds immense significance in the context of T-lineage commitment. From our observations, it appears that epigenetically E2 element is in an open conformation at the DN3 stage, but it is the specific transcription factor occupancy, which is critical in inducing P1 promoter activity at the PTCR-positive stage. As we have shown earlier that NFATc1 nuclear levels increases from DN1 to DN3 stage thus, most likely making it possible to bind to the E2 element and autoregulates its own expression by inducing P1 promoter activity. The elucidation of the signalling pathways regulating $N f a t c 1$ gene regulation will help to better understand $\mathrm{T}$-cell development and will provide insight for therapeutic mani- pulations of NFATc1 activity to achieve immune reconstitution in situations like T-cell lymphopenia (Supplementary Fig. 8c).

The loss of $N f a t c 1$ expression in in vitro cultures though quite intriguing was not due to an enhanced cell death of the immature thymocytes (Supplementary Fig. 4a). Rather, because of very small number of sorted cells their density was too low in the in vitro cultures, which led not only to a lack of interaction between thymocytes and the thymic stroma, but it also resulted in a loss of interaction among thymocytes themselves. In the normal thymus, millions of thymocytes are very densely packed into the available space, which facilitates both thymocyte-stroma and also thymocyte-thymocyte interactions. In our opinion both types of interactions provide the signals essential for $\mathrm{Nfatcl}$ expression. In line with this Nfatc1 expression was not lost when thymocytes were cultured in vitro at a higher density.

We have previously reported a severe $\mathrm{T}$-cell lymphopenia in mice lacking NFATc1 activity in the early thymocytes ${ }^{2}$. Here we show overexpression of NFATc1 also results in T-cell lymphopenia (Fig. 3a-c; Supplementary Fig. 8c), indicating a threshold level of NFATc1 activity is critical for T-cell development. The increase in total NFATc1 activity in the $V a v$-CreNfatc $1 \propto \mathrm{A}^{\mathrm{fl} / \mathrm{fl}}$ mice not only prevented the differentiation of DN3 cells to DN4 stage (Fig. 3d) but it also severely affected several key T-cell-specific genes expression. The dose effect of NFATc1, not only on the DN thymocyte development, but also on the DP to SP differentiation is clearly reflected in the cellularity of the thymus, LNs and spleen in the $V a v$-CreNfatcl $\alpha \mathrm{A}^{\mathrm{fl} / \mathrm{fl}}$ mice compared with WT mice (Fig. 3b,c) underlining the influence of NFATcl activity on thymic T-cell development.

Our results from $\Delta \mathrm{Cam} \times \mathrm{N} 3$ double-tg mice show the tumour suppressor activity of NFATc1 in the experimental setting of hyperactive Notch, as it prevented T-ALL development despite these mice having constitutive Notch3 signalling. In vivo, once the DN3 cells receive pTCR signals they undergo a limited cell cycle and differentiate to DN4 stage (Supplementary Fig. 8c). Unless the pTCR signalling is switched off, it will lead to uncontrolled proliferation resulting in T-ALL development (Supplementary Fig. 8c). To prevent pTCR formation, Ptcra expression needs to be silenced. Here we show that NFATc1 $\alpha$ activity suppresses the Ptcra expression (Fig. 7a-c,e,f). As Nfatcl P1 activity is initiated only at the pTCR-positive thymocytes and pTCR signals induce NFATc1 activation ${ }^{42}$, this is most likely the natural-feedback mechanism to suppress pTCR expression and thereby prevent T-ALL development once the cells have received optimal pTCR signals (Supplementary Fig. 8c). However, loss of function mutations involving NFAT has not been reported in spontaneous cases of either human or murine T-ALL; thus, the tumour suppressive activity of NFATc1 may be specific to the background of the additional oncogenic events. Altogether, our study shows that a threshold level of NFATc1 activity is critical for T-cell development. Any alteration, such as above or below threshold levels of NFATc1 activity will push the delicate balance towards an unwanted extreme, either resulting in T-cell lymphopenia or, likely in combination with other oncogenic events, leading towards the development of leukaemia (Supplementary Fig. 8c).

\section{Methods}

Mice. C57BL/6 WT, calcineurin tg ( $\Delta$ Cam), Rag1-1-, Notch3 tg $(\mathrm{N} 3 \mathrm{tg})$ $\mathrm{Cam} \times \mathrm{N} 3$ tg, Nfatc1 P2 $2^{\mathrm{fl} / \mathrm{fl}}, V a v-\mathrm{CreNfatc1P2} 2^{\mathrm{fl} /+}, V a v-C r e N f a t c 1 P 2^{\mathrm{fl} / \mathrm{fl}}, R 26-c a N-$ fatclaA-Stop ${ }^{\mathrm{f} / \mathrm{fl}}\left(N f a t c 1 \alpha \mathrm{A}^{\mathrm{fl} / \mathrm{ll}}\right), V a v-C r e N f a t c 1 \alpha \mathrm{A}^{\mathrm{fl} /}+$ and $V a v-C r e N f a t c l \alpha \mathrm{A}^{\mathrm{fl} / \mathrm{fl}}$,

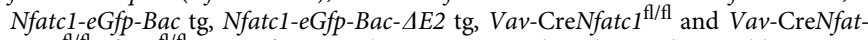
$c 1 \alpha A^{\mathrm{fl} / \mathrm{fl}} N f a t c 1^{\mathrm{fl} / f l}$ mice of 3-8 weeks age were used in this study. Double-mutant mice such as, $V a v$-CreNfatc1P2 $2^{\mathrm{fl} / \mathrm{fl}}, V a v-C r e N f a t c 1 \alpha \mathrm{A}^{\mathrm{fl} / \mathrm{fl}}$ and $V a v-C r e N f a t c 1^{\mathrm{fl} / \mathrm{fl}}$ mice were generated by breeding corresponding floxed mice with $V a v$-Cre tg mice, or by breeding $\Delta \mathrm{Cam}$ with $\mathrm{N} 3 \operatorname{tg}(\Delta \mathrm{Cam} \times \mathrm{N} 3)$ mice, and the triple-mutant 
Vav-CreNfatcl $\alpha A^{\mathrm{fl} / \mathrm{fl}} N f a t c 1^{\mathrm{fl} / \mathrm{fl}}$ mice were generated by crossing Vav-CreNfatc1

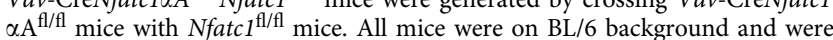
maintained in the animal facility of the Institute of Pathology or in the central animal facility (ZEMM) of University of Wuerzburg, according to the institutional guidelines.

Generation of $\mathbf{N f a t c 1} \mathbf{P} \mathbf{2}^{\mathbf{f l} / \mathbf{f l}}$ mice. To generate the Nfatc1 P2 promoter floxed allele, the $5^{\prime}$ end of the murine NFATc1 cDNA was used to isolate genomic cosmid clones of the Nfatcl gene. The $8.5 \mathrm{~kb}$ XbaI fragment containing the Nfatc1 P2 promoter was identified in DNase I hypersensitive site analysis. The blunt ended left (XbaI to XhoI) and right (XhoI to XbaI) arms were subcloned into the SmaI site of pGL3 basic vector. To incorporate the loxP sites into the left or right arm, loxP sites cut out from the pBS112 SX vector were inserted into the NdeI (448 bp), Eco72I (5,020 bp) and NdeI (7536 bp) sites, respectively. The pTKNeoloxP plasmid containing the neomycin (neo) gene for positive selection and thymidine kinase gene for negative selection was used as basic vector in constructing the targeting vector. Embryonic stem cell clones positive for the targeted P2 floxed allele were selected and were used to generate the chimeric $N f a t c 1 \mathrm{P}^{\mathrm{fl} / \mathrm{fl}}$ mice by blastocyst injection method. Genomic tail DNA from the chimeric mice was analysed by long-distance PCR to confirm the genomic integration of the floxed Nfatc1 P2 allele, and mice were bred with C57BL/6 mice for several generations. WT, Nfatcl $\mathrm{P} 2^{\mathrm{fl} /}+$ and $N f a t c 1 \mathrm{P}^{\mathrm{f}} \mathrm{f}^{\mathrm{fl}}$ mice were genotyped using the following primers: $5^{\prime}$-TCTCCACCTGACTTTCTGTTCC-3' (forward) and rev: $5^{\prime}$-CTCTTCCCAA TGGTTGTCTCTC-3' (reverse).

Generation of Nfatc1-eGfp-Bac- $\Delta$ E2 tg mice. Nfatc1-eGfp-Bac- $\triangle E 2$ construct was generated by deletion of a 1-kb fragment of the $\mathrm{E} 2$ element from the original Nfatcl-eGfp-Bac transgene cassette ${ }^{17}$. The BAC RP23-361H16 containing the murine Nfatcl gene ( $\mathrm{mm} 9$ chr.18, 80.779.051-80.993617, $214 \mathrm{~kb}$ ) was further modified using bacterial homologues recombination to delete $1,000 \mathrm{bp}$ from the intron 10 (mm9 chr.18, 80.809.298-80.808.298, $\Delta \mathrm{E} 2)$. Two homology arms flanking the region of interest were generated by PCR using the primers $5^{\prime}$-targbox-E2_for: $5^{\prime}$-AAGGCGCGCCAAGAGGACCGGAACTCTGTG-3', $5^{\prime}$ targbox-E2_rev: 5'-GCAGTGTCTATGGCTGTGCAAATCACCTG-3', 3 'targ-boxE2_for: $5^{\prime}$-TGCACAGCCATAGACACTGCAAGTCAGGG-3' ${ }^{\prime}$, and $3^{\prime}$ targ-boxE2_rev: 5'-AAGCGGCCGCCTCCACACACACACATATCC- $3^{\prime}$. In a second PCR, the primers $5^{\prime}$ targ-box-E2_for and $3^{\prime}$ targ-box-E2_rev amplified a fusion product that was cloned as an AscI and NotI fragment into the shuttle vector PLD53.RecA. After homologous recombination the linearized BAC DNA was microinjected into blastocysts. Mice tail biopsies were tested by PCR for BAC integration and for deletion of the E2 element with the primers mNc1-DE2-for: $5^{\prime}$-CATACAA ACCCACAAGTGACCA- $3^{\prime}$ and mNc1-DE2-rev: $5^{\prime}$-GAGCCCTACTGAGAG ATGGGAA-3' (522 bp). The $5^{\prime}$-end of integrated BAC DNA was detected with the primer pair $5^{\prime}$ BAC(T7) for: $5^{\prime}$-GGTCCATCCTTTTGTCTCA- $3^{\prime}$ and $3^{\prime} \mathrm{e} 3.6(\mathrm{~T} 7)$ rev: $5^{\prime}$-CGAGCTTGACATTGTAGGA- $3^{\prime}$ (512 bp), and the $3^{\prime}$ end with the primers $5^{\prime}$ e3.6(SP6) for: $5^{\prime}$-CGTCGACATTTAGGTGACA- $3^{\prime}$ and $3^{\prime}$ BAC (SP6) rev: $5^{\prime}$-CCATCGTTCCCTGACTCA-3' (439 bp).

Antibodies and flow cytometry. All Abs used were purchased from BD Pharmingen or from eBioscience: anti-CD4 (GK1.5), anti-CD8 $\alpha$ (53-6.7), anti-CD25 (PC61), anti-CD44 (IM7), anti-CD2 (RM2-5), anti-CD3ع (145-2C11), anti-CD5 (53-7.3), anti-B220 (RA3-6B2), anti-CD11a (2D7), anti-CD11c (N418), anti-CD18 (C71/16), anti-CD24 (M1/69), anti-CD48 (HM48-1), anti-CD49d (R1-2), anti-CD62L (MEL-14), anti-TCR $\beta$ (H57-597), anti-c-Kit (2B8), anti-NK1.1 (PK136), anti-F4/80 (BM8), anti-Gr1 (RB6-8C5), anti-Ter119 (TER-119), anti-cKit (2B8), Annexin V, anti-Bcl-2 (3F11) and Hamster IgG1 Isotype control (A19-3). Biotinylated antibodies were revealed with Streptavidin-Allophycocyanin (APC) or Phycoerythrin-Cy5 (PE-Cy5). For surface staining all primary and secondary antibodies were diluted at 1:300 in PBS containing $0.1 \%$ bovine serum albumin (BSA) and $0.01 \%$ sodium azide. Cell death analysis of DN4 cells cultured overnight in medium was performed by Annexin V (1:100) staining following manufacturer's instruction (BD Pharmingen). Flow cytometry was performed according to standard protocol using FACSCalibur and CellQuest or FlowJo software.

Cell sorting. For FACS sorting of DN1 to DN4 cells, DN thymocytes were enriched by treatment of total thymocytes with biotinylated Abs against CD4 (GK1.5), CD8 (TiB105), NK1.1 (1D4), CD19 (1D3) and MHC class II (2G9) followed by negative selection using Anti-Biotin MicroBeads (Miltenyi Biotec). Purity of DN cells was always $90-95 \%$ as verified by FACS analysis. Enriched DN thymocytes were further stained with APC-conjugated CD4 and CD8, PE-conjugated CD25 and FITC-conjugated CD44 Abs to sort the DN1-DN4 cells gating on the $\mathrm{CD} 4^{-} \mathrm{CD} 8{ }^{-}$population using a FACSVantage or FACSAria (BD Biosciences) flow cytometer.

Intracellular staining. Total thymoctyes of $1 \times 10^{6}$ were first surface stained for CD4, CD8, and CD25 molecules. Afterwards, cells were fixed in $1 \%$ paraformaldehyde ( $15 \mathrm{~min}$ at room temperature) and permeabilized in PBA $\left(1 \times\right.$ PBS, $5 \%$ BSA and $0.02 \% \mathrm{NaN}_{3}$ ) containing $0.5 \%$ saponin (10 min at room temperature). After permeabilization cells were incubated with FITC conjugated mAbs against TCR $\beta$ (1:100), CD3 $\varepsilon$ (1:100), Bcl-2 (1:50) or Hamster IgG1 Isotype control (1:5) diluted in PBA containing 0.5\% saponin for $60 \mathrm{~min}$ at room temperature. Cells were washed twice in $1 \mathrm{ml}$ of $\mathrm{PBA} / 0.5 \%$ saponin, once with $\mathrm{PBS} / 0.1 \% \mathrm{BSA} / 0.01 \%$ azide and analysed by FACS.

Immunofluorescence staining and ChIP assays. Sorted WT DN1 + DN2 + DN3 or DN4 cells were fixed in $0.5 \%$ formaldehyde, followed by cytospin on to glass slides at 300 r.p.m. for $3 \mathrm{~min}$. Cells were incubated with anti-NFATc1 $\alpha$ (ImmunoGlobe; IG-457), followed by Alexa Fluor 555-conjugated donkey anti-rabbit IgG (A-31572; Molecular Probes) and DAPI (4,6-diamidino-2-phenylindole; Molecular Probes). Image acquisition and analysis were done with a TCS SP2 Leica confocal microscope and software.

ChIP assays on WT DN thymocytes, or Rag1 ${ }^{-1-}$ and $\Delta$ Cam DN3 cells with anti-NFATc1 (Santacruz; sc-7294), anti-NFATc1 $\alpha$, antiRBPJк (Cell Signaling; 5313) and anti-IкB- $\alpha$ (Santa Cruz; sc-371) were done as described previously ${ }^{2}$. Briefly, $1 \times 10^{7}$ freshly isolated DN thymocytes from WT, or DN3 cells from Rag1 $^{-1-}$ and $\Delta$ Cam tg mice were cross-linked using $1 \%$ formaldehyde. Isolated nuclei were sonicated so that the average length of chromosomal DNA became 500-750 bp. Chromatin solution was precleared with Sepharose G, and chromatin was immunoprecipitated by incubating with $6-8 \mu \mathrm{g}$ of each antibody mentioned above for overnight at $4{ }^{\circ} \mathrm{C}$. Immune complexes were collected on protein G-Sepharose beads and immunoprecipitates were eluted with $1 \%$ SDS, $50 \mathrm{mM}$ $\mathrm{NaHCO}_{3}$. After reversal of cross-links and deproteination, purified DNA was used for amplification of the Ptcra promoter region bound to NFATc1, NFATc1 $\alpha$, and RBPJ $\kappa$ with the following primers: $5^{\prime}$ - AGGGAACAGAATTCAAGGCTG- $3^{\prime}$ (forward) and $5^{\prime}$-ATCTTCTTGCTCTAAATCTCC-3' (reverse).

ChIP-Seq and RNA-Seq analysis. ChIP-seq ${ }^{43}$ and Small RNA-seq ${ }^{8}$ were performed as described previously using same standards and quality controls.

ChIP-Seq. Briefly, DN cells were isolated from Bl.6 Rag2 $2^{-/-}$mice and crosslinked with $1 \%$ formaldehyde. Histone ChIPs were performed by using crosslinked nuclear extracts from 5 million cells with $2 \mu \mathrm{g}$ specific antibodies and $20 \mu \mathrm{l}$ Dynabeads Protein G suspension (Life Technologies, USA), whereas 50 million cells were used for Pol II ChIPs. Antibodies used are described in Koch et al., with the exception of H3K27ac (ab4729; Abcam). DNA was extracted from immunoprecipitated chromatin and was used to prepare sequencing library using TrueSeq ChIP-seq Library Preparation Kit (Illumina Inc., USA) according to manufacturer's instructions, and sequenced on a Genome Analyzer II sequencing platform (Illumina Inc., USA). Data processing, including input subtraction and wig files generation was performed as previously described ${ }^{43}$.

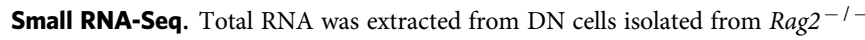
cells using TriZol Reagent (Life Technologies, USA). Total RNA of $10 \mu \mathrm{g}$ was fractionated by TBE-Urea PAGE to isolate RNA fragments ranging from 15 nucleotides to 70 nucleotides length. These size-selected small RNAs were used to prepare sequencing library using Small RNA-Seq Library Preparation Kit (Illumina Inc., USA) according to manufacturer's instructions. Libraries were then sequenced on a Genome Analyzer II sequencing platform (Illumina Inc., USA). The RNA-Seq data used is accessible in gene omnibus under the accession number GSE44578.

OP9-DL1 co-culture assays and in vitro cultures. $4 \times 10^{4}$ sorted WT or VavCreNfatc1 $\alpha \mathrm{A}^{\mathrm{f} / \mathrm{fl}} \mathrm{DN1}$ - DN4 cells were co-cultured on monolayers of OP9 stromal cells expressing the Notch ligand delta-like 1 (OP9-DL1) in X-vivo 20 medium supplemented with rhFLT3 ligand $\left(5 \mathrm{ng} \mathrm{ml}^{-1}\right)$ and $\operatorname{rhIL}-7\left(1 \mathrm{ng} \mathrm{ml}^{-1}\right)$ for 4 days. Subsequently, thymocytes were analysed for differentiation into DP cells by flow cytometry. In vitro cultures of sorted DN1-DN4 cell populations or $\mathrm{CD} 4{ }^{+} \mathrm{T}$ cells were performed either in medium (RPMI-1640, 10\% FCS) only or with CsA $\left(100 \mathrm{ng} \mathrm{ml}^{-1}\right)$, 8-CPT-cAMP $(50 \mu \mathrm{M})$, Forskolin (Fsk; $\left.100 \mu \mathrm{M}\right), \mathrm{CD} 48(5$ or $0.5 \mu \mathrm{g} \mathrm{ml}^{-1}$ ), PMA $\left(100 \mathrm{ng} \mathrm{ml}^{-1}\right.$ ), Fibronectin (Fibr; $1 \mu \mathrm{g} \mathrm{ml}^{-1}$ ) supplemented with rhIL-7 $\left(10 \mathrm{ng} \mathrm{ml}^{-1}\right)$.

Semiquantitative RT-PCR. For RT-PCR, cDNA was synthesized from cells either freshly isolated or cultured as indicated in each case using Miltenyi Biotech cDNA synthesis kit. To check CD2-CD48 signalling-induced Nfatc1 expression, $1 \times 10^{6}$ sorted WT DN3 cells were cultured in cRPMI- 1640 medium ( $10 \%$ fetal calf serum) supplemented with $10 \mathrm{ng} \mathrm{ml}^{-1}$ rhIL-7. Cells were either left unstimulated or stimulated with plate bound CD48 antibodies ( $5 \mu \mathrm{g}$ ), PMA (100 ng) or with $\mathrm{CD} 48+$ PMA for $12 \mathrm{~h}$ at $37^{\circ} \mathrm{C}$. Similarly, $8 \times 10^{6} \mathrm{CD} 4^{+} \mathrm{T}$ cells from lymph nodes of WT mice were left unstimulated or stimulated with plate bound Fibronectin $(1 \mu \mathrm{g}), \mathrm{CD} 48(0.5 \mu \mathrm{g})$ or Fibronectin $+\mathrm{CD} 48$ for $24 \mathrm{~h}$ at $37^{\circ} \mathrm{C}$. Semiquantitative RT-PCR was performed to check the levels of expression of indicated genes. $\beta$-actin expression levels were used as control. RT-PCRs to check the expression of six Nfatcl isoform were performed using the Fermentas Long distance PCR Kit 
(Cat. \# K0181). Primer sequences are available (Supplementary Table 1) in the Supplemental Information online.

Methylation analysis. Genomic DNA was isolated from FACS sorted WT DN3 and DN4 cells following standard protocol. Sodium bisulphite treatment of genomic DNA was performed using ZYMO Research EZ DNA Methylation Gold kit (Cat. \# D5005) according to manufacturer's instructions. All PCR were performed on MWG AG Biotech Primus 96 plus thermocyclers (Ebersberg, Germany) in a final volume of $25 \mu$ lincluding $1 \times$ PCR Master Mix (Thermo Scientific, Cat. \# K0171), 10 pmol each of forward and reverse primers (Nfatc1 P1d forward: (-1297 -818) 5'-GGAGATTTGAAAGAGAAAAA-3' and Nfatc1 P1d reverse: $(-1297-818) 5^{\prime}$-AAARCTACTCTCCCTTTTAAT- $\left.3^{\prime}\right)$, and $10 \mathrm{ng}$ of bisulphite-treated genomic DNA. The following amplification protocol was used: $95^{\circ} \mathrm{C}$ for $10 \mathrm{~min}$ and 40 cycles of $95^{\circ} \mathrm{C}$ for $1 \mathrm{~min}, 52^{\circ} \mathrm{C}$ for $45 \mathrm{~s}$, and $72^{\circ} \mathrm{C}$ for $1 \mathrm{~min}$, and a final amplification step of $72^{\circ} \mathrm{C}$ for $10 \mathrm{~min}$. PCR products were eluted and purified using innuPREP DOUBLEpure kit (Analytik Jena AG, Cat. \# $845-\mathrm{KS}-5050050$ ) and cloned into a pGEM T-Easy Vector (Promega, Cat. \# A1360) according to manufacturer's protocol. E. coli (Top-F) were transformed with plasmid ligates and were selected by $a m p^{R}$ on Lurient-Broth Agar plates. Colonies expressing the full-size amplificate were detected by blue-white selection and DNA was prepared according to standard protocols. Positive clones were further determined by restriction digestion (EcoRI) and subsequently sequenced. PCRs were performed with primers for T7 and SP6 (Sigma-Aldrich) in both $5^{\prime}$ and $3^{\prime}$ direction using Big Dye Terminator v3.1 Cycle Sequencing Kit (Applied Biosystems, Product $\mathrm{P} / \mathrm{N}$ 4336935). Sequence data was generated with an ABI Prism 3130xl, and analysed with BiQ Analyzer v2.0 (Christoph Bock).

Immunoblotting. Positively selected $\mathrm{LN} \mathrm{CD} 4^{+} \mathrm{T}$ cells $\left(2 \times 10^{7}\right)$ from Nfatc1$e G f p-B a c$ tg or Nfatc1-eGfp-Bac- $\Delta E 2$ tg reporter mice were left unstimulated or stimulated with aCD3 $\left(3 \mu \mathrm{g} \mathrm{ml}^{-1}\right)$ plus aCD28 $\left(5 \mu \mathrm{g} \mathrm{ml}^{-1}\right)$ for $48 \mathrm{~h}$. Subsequently, whole-cell protein extracts were prepared and $20 \mu \mathrm{g}$ protein from each sample was subjected to immunoblotting in $12 \%$ polyacrylamide gels. Proteins were transferred to a nitrocellulose membrane followed by immunodetection of GFP with Abs directed against GFP (Santacruz; sc-9996), or NFATcl $\alpha$ with Abs raised against the $\alpha$-peptide of NFATc1 (ImmunoGlobe; IG-457). Images in Fig. $6 \mathrm{~d}$ are cropped for presentation excluding the lanes 3 and 6, which represents B-cell extracts. Full-size images from original scan with molecular weight marker are presented in Supplementary Fig. 7.

Luciferase reporter assays. One microgram of a murine Ptcra luciferase reporter construct containing $400 \mathrm{bp}$ enhancer region ( $-4,400$ to $4,000 \mathrm{bp}$ ) and $1.1 \mathrm{~kb}$ DNA fragment spanning murine Ptcra promoter region $(-1034$ to $+66 \mathrm{bp})$, or $1 \mu \mathrm{g}$ of a luciferase reporter construct containing $293 \mathrm{bp}$ DNA fragment spanning murine proximal $\mathrm{Il} 2$ promoter region was co-transfected along with $4 \mu \mathrm{g}$ control vectors into EL-4 thymoma cells by DEAE/Dextran method. Thirty six hours post-transfection cells were left unstimulated or stimulated with PMA plus Ionomycin ( $100 \mathrm{ng} \mathrm{ml}^{-1}$ each, Calbiochem) for $12 \mathrm{~h}$.

Afterwards, luciferase activity representing the Ptcra promoter transactivation was measured using a MicroLumat LB 96P (EG\&G Berthold) luminometer. For Nfatc1 P1 and P2 promoter constructs, a 1,430 bp (-1155 to + 275) DNA fragment in the region around exon 1 , and a $1260 \mathrm{bp}(-1205$ to +55$)$ DNA fragment in the region around exon 2, respectively, were cloned as NheI/Xhol-digested PCR products into the pGl3basic vector. The potential enhancer elements E1-E4 were cloned $3^{\prime}$ of the luciferase reporter gene as SalI-digested PCR products. Mutations of potential NFAT, GATA and PU.1 binding sites in the E2 element were introduced by site-directed mutagenesis. The details about the NFATc1 (Nc1A) and Notch1 intracellular domain (N1-IC) expression constructs have been described previously ${ }^{5,44}$. To assess the effects of NFATc1 activity on Notchinduced Ptcra promoter activation, $1 \times 10^{6}$ Jurkat cells were transfected by the ViaFect Transfection Reagent (Promega, Wisconsin, USA) with $5 \mu \mathrm{g}$ DNA containing all necessary constructs according to the manufacturer's protocol. Twenty four hours later fresh medium was added and the cells were divided into untreated or ionomycin (I) treated samples. After further $16 \mathrm{~h}$ cells were collected to estimate luciferase activity.

Data availability. ChIP-Seq (accession numbers GSE80138 and GSE55635) and RNA-Seq (accession number GSE44578) data are available in the gene omnibus database. All other data supporting the findings of this study are available with the article, and can also be obtained from the authors.

\section{References}

1. Godfrey, D. I., Kennedy, J., Suda, T. \& Zlotnik, A. A developmental pathway involving four phenotypically and functionally distinct subsets of CD3-CD4CD8- triple-negative adult mouse thymocytes defined by CD44 and CD25 expression. J. Immunol. 150, 4244-4252 (1993).

2. Patra, A. K. et al. An alternative NFAT-activation pathway mediated by IL-7 is critical for early thymocyte development. Nat. Immunol. 14, 127-135 (2013).
3. Xanthoudakis, S. et al. An enhanced immune response in mice lacking the transcription factor NFAT1. Science 272, 892-895 (1996).

4. Ranger, A. M., Oukka, M., Rengarajan, J. \& Glimcher, L. H. Inhibitory function of two NFAT family members in lymphoid homeostasis and Th2 development. Immunity 9, 627-635 (1998).

5. Chuvpilo, S. et al. Autoregulation of NFATc1/A expression facilitates effector T cells to escape from rapid apoptosis. Immunity 16, 881-895 (2002).

6. Chuvpilo, S. et al. Multiple NF-ATc isoforms with individual transcriptional properties are synthesized in T lymphocytes. J. Immunol. 162, 7294-7301 (1999).

7. Serfling, E., Chuvpilo, S., Liu, J., Hofer, T. \& Palmetshofer, A. NFATc1 autoregulation: a crucial step for cell-fate determination. Trends. Immunol. 27, 461-469 (2006).

8. Lepoivre, C. et al. Divergent transcription is associated with promoters of transcriptional regulators. BMC Genomics 14, 914 (2013).

9. Patra, A. K. et al. PKB rescues calcineurin/NFAT-induced arrest of Rag expression and pre-T cell differentiation. J. Immunol. 177, 4567-4576 (2006)

10. Bellavia, D. et al. Constitutive activation of NF-kappaB and T-cell leukaemia/ lymphoma in Notch3 transgenic mice. EMBO J. 19, 3337-3348 (2000).

11. de Boer, J. et al. Transgenic mice with hematopoietic and lymphoid specific expression of Cre. Eur. J. Immunol. 33, 314-325 (2003).

12. Baumgart, S. et al. Inflammation-induced NFATc1-STAT3 transcription complex promotes pancreatic cancer initiation by KrasG12D. Cancer Discov. 4, 688-701 (2014).

13. Porritt, H. E. et al. Heterogeneity among DN1 prothymocytes reveals multiple progenitors with different capacities to generate $\mathrm{T}$ cell and non- $\mathrm{T}$ cell lineages. Immunity 20, 735-745 (2004).

14. Bopp, T. et al. Cyclic adenosine monophosphate is a key component of regulatory T cell-mediated suppression. J. Exp. Med. 204, 1303-1310 (2007).

15. Gavin, M. A. et al. Foxp3-dependent programme of regulatory T-cell differentiation. Nature 445, 771-775 (2007).

16. Conche, C., Boulla, G., Trautmann, A. \& Randriamampita, C. T cell adhesion primes antigen receptor-induced calcium responses through a transient rise in adenosine 3',5'-cyclic monophosphate. Immunity 30, 33-43 (2009).

17. Hock, M. et al. NFATc1 induction in peripheral T and B lymphocytes. J. Immunol. 190, 2345-2353 (2013).

18. Meuer, S. C. et al. An alternative pathway of T-cell activation: a functional role for the $50 \mathrm{kd}$ T11 sheep erythrocyte receptor protein. Cell 36, 897-906 (1984).

19. Yang, S. Y., Chouaib, S. \& Dupont, B. A common pathway for T lymphocyte activation involving both the CD3-Ti complex and CD2 sheep erythrocyte receptor determinants. J. Immunol. 137, 1097-1100 (1986).

20. Kato, K. et al. CD48 is a counter-receptor for mouse CD2 and is involved in T cell activation. J. Exp. Med. 176, 1241-1249 (1992).

21. Kaplan, A. J. et al. Production and characterization of soluble and transmembrane murine CD2. Demonstration that CD48 is a ligand for CD2 and that CD48 adhesion is regulated by CD2. J. Immunol. 151, 4022-4032 (1993).

22. Zhu, B., Davies, E. A., van der Merwe, P. A., Calvert, T. \& Leckband, D. E. Direct measurements of heterotypic adhesion between the cell surface proteins CD2 and CD48. Biochemistry 41, 12163-12170 (2002).

23. Arulanandam, A. R. et al. A soluble multimeric recombinant $\mathrm{CD} 2$ protein identifies CD48 as a low affinity ligand for human CD2: divergence of CD2 ligands during the evolution of humans and mice. J. Exp. Med. 177, 1439-1450 (1993).

24. Carrera, A. C., Rincon, M., De Landazuri, M. O. \& Lopez-Botet, M. CD2 is involved in regulating cyclic AMP levels in T cells. Eur. J. Immunol. 18, 961-964 (1988).

25. Hahn, W. C., Rosenstein, Y., Burakoff, S. J. \& Bierer, B. E. Interaction of CD2 with its ligand lymphocyte function-associated antigen-3 induces adenosine 3', $5^{\prime}$-cyclic monophosphate production in T lymphocytes. J. Immunol. 147, 14-21 (1991)

26. Guyot, D. J., Newbound, G. C. \& Lairmore, M. D. CD2 signalling induces phosphorylation of CREB in primary lymphocytes. Immunology 95, 544-552 (1998).

27. Barton, K. et al. Defective thymocyte proliferation and IL-2 production in transgenic mice expressing a dominant-negative form of CREB. Nature 379, 81-85 (1996).

28. Rudolph, D. et al. Impaired fetal $\mathrm{T}$ cell development and perinatal lethality in mice lacking the cAMP response element binding protein. Proc. Natl Acad. Sci. USA 95, 4481-4486 (1998).

29. Hunig, T., Tiefenthaler, G., Meyer zum Buschenfelde, K. H. \& Meuer, S. C. Alternative pathway activation of $\mathrm{T}$ cells by binding of $\mathrm{CD} 2$ to its cell-surface ligand. Nature 326, 298-301 (1987).

30. Killeen, N., Stuart, S. G. \& Littman, D. R. Development and function of T cells in mice with a disrupted CD2 gene. EMBO J. 11, 4329-4336 (1992).

31. Teh, S. J., Killeen, N., Tarakhovsky, A., Littman, D. R. \& Teh, H. S. CD2 regulates the positive selection and function of antigen-specific CD4- CD8 + T cells. Blood 89, 1308-1318 (1997). 
32. Evans, C. F., Rall, G. F., Killeen, N., Littman, D. \& Oldstone, M. B. CD2deficient mice generate virus-specific cytotoxic $\mathrm{T}$ lymphocytes upon infection with lymphocytic choriomeningitis virus. J. Immunol. 151, 6259-6264 (1993).

33. Kyewski, B. A. et al. The effects of anti-CD2 antibodies on the differentiation of mouse thymocytes. Eur. J. Immunol. 19, 951-954 (1989).

34. Gonzalez-Cabrero, J. et al. CD48-deficient mice have a pronounced defect in CD4(+) T cell activation. Proc. Natl Acad. Sci. USA 96, 1019-1023 (1999).

35. Paganin, M. \& Ferrando, A. Molecular pathogenesis and targeted therapies for NOTCH1-induced T-cell acute lymphoblastic leukaemia. Blood Rev. 25, 83-90 (2011).

36. Weng, A. P. et al. Activating mutations of NOTCH1 in human T cell acute lymphoblastic leukaemia. Science 306, 269-271 (2004).

37. Ferrando, A. A. The role of NOTCH1 signaling in T-ALL. Hematol. Am. Soc. Hematol. Educ. Program 353-361 (2009).

38. Bellavia, D. et al. Combined expression of pTalpha and Notch3 in T cell leukaemia identifies the requirement of preTCR for leukemogenesis. Proc. Natl Acad. Sci. USA 99, 3788-3793 (2002).

39. O’Neil, J. et al. Activating Notch1 mutations in mouse models of T-ALL. Blood 107, 781-785 (2006).

40. Talora, C. et al. Cross talk among Notch3, pre-TCR, and Tal1 in T-cell development and leukemogenesis. Blood 107, 3313-3320 (2006).

41. Reizis, B. \& Leder, P. Direct induction of T lymphocyte-specific gene expression by the mammalian Notch signaling pathway. Genes Dev. 16, 295-300 (2002).

42. Aifantis, I., Gounari, F., Scorrano, L., Borowski, C. \& von Boehmer, H. Constitutive pre-TCR signaling promotes differentiation through $\mathrm{Ca} 2+$ mobilization and activation of NF-kappaB and NFAT. Nat. Immunol. 2, 403-409 (2001).

43. Koch, F. et al. Transcription initiation platforms and GTF recruitment at tissuespecific enhancers and promoters. Nat. Struct. Mol. Biol. 18, 956-963 (2011).

44. Capobianco, A. J., Zagouras, P., Blaumueller, C. M., Artavanis-Tsakonas, S. \& Bishop, J. M. Neoplastic transformation by truncated alleles of human NOTCH1/TAN1 and NOTCH2. Mol. Cell. Biol. 17, 6265-6273 (1997).

\section{Acknowledgements}

We thank C. Linden (University of Wuerzburg) for excellent FACS sorting, Ilona Pietrowski and Doris Michel for luciferase reporter assays, D. Kioussis (Medical Research Council, National Institute for Medical Research, London) for Vav-Cre mice, J. Liu (University of Wuerzburg), A. Waisman and K. Reifenberg (University of Mainz) for help in generating $N f a t c 1 P 2^{\mathrm{fl} / \mathrm{fl}}$ and $N f a t c 1-e G f p-B a c-\Delta E 2$ tg mice. This work was supported by an 'EMBO Short Term Fellowship' (ASTF No: 93-06), and a 'PostDoc Plus Funding' grant (Graduate School of Life Sciences; GSLS, University of Wuerzburg) to A.K.P., and by the Deutsche Forschungsgemeinschaft (DFG) grants TRR52 (S.K.H. and E.S.) and the Wilhelm-Sander Foundations (E.S.).

\section{Author contributions}

S.K.H. generated the Nfatc1-eGfp-Bac- $\Delta E 2 \mathrm{tg}$ mice and performed all E2 enhancer related studies; R.R. did the methylation analysis and analysed the Nfatc1-eGfp-Bac- $\Delta E 2$ transgenic mice; K.M. performed the ChIP assays; K.P.K. contributed in generating the NfatclP2 $2^{\mathrm{f} / \mathrm{fl}}$ mice; M.A.M, P.C. and J.C.A. performed the ChIP-Seq and RNA-Seq analysis; A.A. assisted in OP9 and OP9-DL1 co-culture experiments, and establishing the $N f a t c 1 P 2^{\mathrm{fl} / \mathrm{fl}}$ mice line; V.E. generated the ROSA26-caNfatcl $\alpha A$-Stop ${ }^{\mathrm{fl} / \mathrm{fl}}$ mice and, C.T. and I.S. contributed with $\mathrm{N} 3$ tg mice and Jurkat cell line related experiments. E.S. contributed to several aspects of this study and wrote the manuscript. A.K.P. conceived the project, designed and performed majority experiments and wrote the manuscript.

\section{Additional information}

Supplementary Information accompanies this paper at http://www.nature.com/ naturecommunications

Competing financial interests: The authors declare no competing financial interests.

Reprints and permission information is available online at http://npg.nature.com/ reprintsandpermissions/

How to cite this article: Klein-Hessling, S. et al. A threshold level of NFATc1 activity facilitates thymocyte differentiation and opposes notch-driven leukaemia development. Nat. Commun. 7:11841 doi: 10.1038/ncomms11841 (2016).

This work is licensed under a Creative Commons Attribution 4.0 International License. The images or other third party material in this article are included in the article's Creative Commons license, unless indicated otherwise in the credit line; if the material is not included under the Creative Commons license, users will need to obtain permission from the license holder to reproduce the material. To view a copy of this license, visit http://creativecommons.org/licenses/by/4.0/ 